\title{
Forced vibrations of wave equations with non-monotone nonlinearities
}

\section{Vibrations forcées d'équations des ondes avec des non-linéarités non-monotones}

\author{
Massimiliano Berti ${ }^{a}$, Luca Biasco ${ }^{\text {b,* }}$ \\ a SISSA, via Beirut 2-4, Trieste, Italy \\ b Università di Roma 3, Largo S. Leonardo Murialdo, Roma, Italy
}

Received 15 December 2004; received in revised form 11 May 2005; accepted 20 May 2005

Available online 19 January 2006

\begin{abstract}
We prove existence and regularity of periodic in time solutions of completely resonant nonlinear forced wave equations with Dirichlet boundary conditions for a large class of non-monotone forcing terms. Our approach is based on a variational LyapunovSchmidt reduction. It turns out that the infinite dimensional bifurcation equation exhibits an intrinsic lack of compactness. We solve it via a minimization argument and a-priori estimate methods related to the regularity theory of [P. Rabinowitz, Periodic solutions of nonlinear hyperbolic partial differential equations, Comm. Pure Appl. Math. 20 (1967) 145-205].

(C) 2006 L'Association Publications de l'Institut Henri Poincaré. Published by Elsevier B.V. All rights reserved
\end{abstract}

\section{Résumé}

On prouve l'existence et la régularité de solutions périodiques en temps d'équations des ondes non linéaires forcées, complètement résonnantes, avec des conditions au bord de Dirichlet, pour une grande classe de termes forcants non-monotones. Notre approche est basée sur une réduction de Lyapunov-Schmidt variationnelle. L'équation de bifurcation en dimension infinie présente un manque intrinsèque de compacité. Nous la résolvons par un argument de minimisation et à l'aide d'estimations a priori inspirées de la théorie de la régularité de [P. Rabinowitz, Periodic solutions of nonlinear hyperbolic partial differential equations, Comm. Pure Appl. Math. 20 (1967) 145-205].

(C) 2006 L'Association Publications de l'Institut Henri Poincaré. Published by Elsevier B.V. All rights reserved

MSC: 35L05; 35L20; 35B10; 37K10

Keywords: Wave equation; Periodic solutions; Variational methods; A-priori estimates; Lyapunov-Schmidt reduction

\footnotetext{
* Corresponding author. Tel.: +39040 3787449; fax +39040 3787528.

E-mail addresses: berti@sissa.it (M. Berti), biasco@mat.uniroma3.it (L. Biasco).
} 


\section{Introduction}

In this paper we consider the problem of finding nontrivial time-periodic solutions of the completely resonant nonlinear forced wave equation

$$
\square u=\varepsilon f(t, x, u ; \varepsilon)
$$

with Dirichlet boundary conditions

$$
u(t, 0)=u(t, \pi)=0
$$

where $\square:=\partial_{t t}-\partial_{x x}$ is the D'Alembertian operator, $\varepsilon$ is a small parameter and the nonlinear forcing term $f(t, x, u ; \varepsilon)$ is $T$-periodic in time. We consider the case when $T$ is a rational multiple of $2 \pi$ and, for simplicity of exposition, we shall assume

$$
T=2 \pi \text {. }
$$

We look for nontrivial $2 \pi$-periodic in time solutions $u(t, x)$ of $(1.1),(1.2)$, i.e. satisfying

$$
u(t+2 \pi, x)=u(t, x) .
$$

For $\varepsilon=0,(1.1),(1.2)$ reduces to the linear homogeneous wave equation

$$
\left\{\begin{array}{l}
\square u=0, \\
u(t, 0)=u(t, \pi)=0
\end{array}\right.
$$

which possesses an infinite dimensional space of solutions which are $2 \pi$-periodic in time and of the form $v(t, x)=$ $\hat{v}(t+x)-\hat{v}(t-x)$ for any $2 \pi$-periodic function $\hat{v}(\cdot)$. For this reason equation (1.1), (1.2) is called completely resonant.

The main difficulty for proving existence of solutions of (1.1)-(1.3) for $\varepsilon \neq 0$ is to find from which periodic orbits of the linear equation (1.4) the solutions of the nonlinear equation (1.1) branch off. This requires to solve an infinite dimensional bifurcation equation (also called kernel equation) with an intrinsic lack of compactness.

The first breakthrough regarding problem (1.1)-(1.3) was achieved by Rabinowitz in [18] where existence and regularity of solutions was proved for nonlinearities satisfying the strongly monotone assumption $\left(\partial_{u} f\right)(t, x, u) \geqslant$ $\beta>0$. Using methods inspired by the theory of elliptic regularity, [18] proved the existence of a unique curve of smooth solutions for $\varepsilon$ small. Other existence results of weak and classical solutions have been obtained, still in the strongly monotone case, in $[10,12,16]$.

Subsequently, Rabinowitz [19] was able to prove existence of weak solutions of (1.1)-(1.3) for a class of weakly monotone nonlinearities like $f(t, x, u)=u^{2 k+1}+G(t, x, u)$ where $G\left(t, x, u_{2}\right) \geqslant G\left(t, x, u_{1}\right)$ if $u_{2} \geqslant u_{1}$. Actually, in [19] bifurcation of a global continuum branch of weak solutions is proved. For other local existence results in the weakly monotone case we mention $[14,20]$.

In all the quoted papers the monotonicity assumption (strong or weak) is the key property for overcoming the lack of compactness in the infinite dimensional kernel equation.

We underline that, in general, the weak solutions obtained in [19] are only continuous functions. Concerning regularity, Brezis and Nirenberg [10] proved - but only for strongly monotone nonlinearities - that any $L^{\infty}$-solution of (1.1)-(1.3) is smooth, even in the nonperturbative case $\varepsilon=1$, whenever the nonlinearity $f$ is smooth.

On the other hand, very little is known about existence and regularity of solutions if we drop the monotonicity assumption on the forcing term $f$. Willem [21], Hofer [15] and Coron [11] have considered the class of equations (1.1), (1.2) where $f(t, x, u)=g(u)+h(t, x), \varepsilon=1$, and $g(u)$ satisfies suitable linear growth conditions. Existence of weak solutions is proved, in [15,21], for a set of $h$ dense in $L^{2}$, although explicit criteria that characterize such $h$ are not provided. The infinite dimensional bifurcation problem is overcome by assuming non-resonance hypotheses between the asymptotic behavior of $g(u)$ and the spectrum of $\square$. On the other side, Coron [11] finds weak solutions assuming the additional symmetry $h(t, x)=h(t+\pi, \pi-x)$ and restricting to the space of functions satisfying $u(t, x)=u(t+\pi, \pi-x)$, where the Kernel of the d'Alembertian operator $\square$ reduces to 0. Actually [11] also deals with the autonomous case $h \equiv 0$, proving for the first time existence of nontrivial solutions for non-monotone nonlinearities. For some more recent results see for example [3].

In the present paper we prove existence and regularity of solutions of (1.1)-(1.3) for a large class of non-monotone forcing terms $f(t, x, u)$, including, for example, 


$$
\begin{aligned}
& f(t, x, u)= \pm u^{2 k}+h(t, x), \quad \text { see Theorem } 1 ; \\
& f(t, x, u)= \pm u^{2 k}+u^{2 k+1}+h(t, x), \quad \text { see Theorem } 2 ; \\
& f(t, x, u)= \pm u^{2 k}+\tilde{f}(t, x, u) \quad \text { with }\left(\partial_{u} \tilde{f}\right)(t, x, u) \geqslant \beta>0, \quad \text { see Theorem } 3 .
\end{aligned}
$$

The precise results were announced in [4] and will be stated in the next Subsection 1.1, see Theorems 1, 2 and 3. Their proof is based on a variational Lyapunov-Schmidt reduction, minimization arguments and a-priori estimate methods inspired to regularity theory of [18]. We anticipate that our approach - explained in Subsection 1.2 - is not merely a sharpening of the ideas of $[18,19]$, which, to deal with non-monotone nonlinearities, require a significant change of perspective.

We mention that in the last years several results on bifurcation of free vibrations for completely resonant autonomous wave equations have been proved in $[2,5-8,13]$. The main differences with respect to the present case are that: a "small divisor" problem in solving the "range equation" appears (here no small divisor problem is present due to the assumption $T=2 \pi$, see Remark 1.7), but the infinite dimensional "bifurcation equation" - whose solutions is the main problem of the present paper - gains crucial compactness properties, see Remark 1.8 .

\subsection{Main results}

We look for solutions $u: \Omega \rightarrow \mathbb{R}$ of (1.1) in the Banach space

$$
E:=H^{1}(\Omega) \cap C_{0}^{1 / 2}(\bar{\Omega}), \quad \Omega:=\mathbb{T} \times(0, \pi),
$$

where $H^{1}(\Omega)$ is the usual Sobolev space and $C_{0}^{1 / 2}(\bar{\Omega})$ is the space of all the $1 / 2$-Hölder continuous functions $u: \bar{\Omega} \rightarrow \mathbb{R}$ satisfying (1.2), endowed with norm ${ }^{1}$

$$
\|u\|_{E}:=\|u\|_{H^{1}(\Omega)}+\|u\|_{C^{1 / 2}(\bar{\Omega})}
$$

Critical points of the Lagrangian action functional $\Psi \in C^{1}(E, \mathbb{R})$

$$
\Psi(u):=\Psi(u, \varepsilon):=\int_{\Omega}\left[\frac{u_{t}^{2}}{2}-\frac{u_{x}^{2}}{2}+\varepsilon F(t, x, u ; \varepsilon)\right] \mathrm{d} t \mathrm{~d} x,
$$

where $F(t, x, u ; \varepsilon):=\int_{0}^{u} f(t, x, \xi ; \varepsilon) \mathrm{d} \xi$, are weak solutions of (1.1)-(1.3).

For $\varepsilon=0$, the critical points of $\Psi$ in $E$ reduce to the solutions of the linear equation (1.4) and form the subspace $V:=N \cap H^{1}(\Omega)$ where

$$
N:=\left\{v(t, x)=\hat{v}(t+x)-\hat{v}(t-x) \mid \hat{v} \in L^{2}(\mathbb{T}) \text { and } \int_{0}^{2 \pi} \hat{v}(s) \mathrm{d} s=0\right\} .
$$

Note that $V:=N \cap H^{1}(\Omega)=\left\{v(t, x)=\hat{v}(t+x)-\hat{v}(t-x) \in N \mid \hat{v} \in H^{1}(\mathbb{T})\right\} \subset E$, since any function $\hat{v} \in H^{1}(\mathbb{T})$ is 1/2-Hölder continuous.

Let $N^{\perp}:=\left\{h \in L^{2}(\Omega) \mid \int_{\Omega} h v=0, \forall v \in N\right\}$ denote the $L^{2}(\Omega)$-orthogonal of $N$.

We prove the following theorem:

Theorem 1. Let $f(t, x, u)=\beta u^{2 k}+h(t, x)$ and $h \in N^{\perp}$ satisfies $h(t, x)>0($ or $h(t, x)<0)$ a.e. in $\Omega$. Then, for $\varepsilon$ small enough, there exists at least one weak solution $u \in E$ of (1.1)-(1.3) with $\|u\|_{E} \leqslant C|\varepsilon|$. If, moreover, $h \in$ $H^{j}(\Omega) \cap C^{j-1}(\bar{\Omega}), j \geqslant 1$, then $u \in H^{j+1}(\Omega) \cap C_{0}^{j}(\bar{\Omega})$ with $\|u\|_{H^{j+1}(\Omega)}+\|u\|_{C^{j}(\bar{\Omega})} \leqslant C|\varepsilon|$ and therefore, for $j \geqslant 2, u$ is a classical solution.

\footnotetext{
$\overline{{ }^{1} \text { Here }\|u\|_{H^{1}(\Omega)}^{2}}:=\|u\|_{L^{2}(\Omega)}^{2}+\left\|u_{x}\right\|_{L^{2}(\Omega)}^{2}+\left\|u_{t}\right\|_{L^{2}(\Omega)}^{2}$ and$$
\|u\|_{C^{1 / 2}(\bar{\Omega})}:=\|u\|_{C^{0}(\Omega)}+\sup _{(t, x) \neq\left(t_{1}, x_{1}\right)} \frac{\left|u(t, x)-u\left(t_{1}, x_{1}\right)\right|}{\left(\left|t-t_{1}\right|+\left|x-x_{1}\right|\right)^{1 / 2}} .
$$ 
Theorem 1 is a corollary of the following more general result which enables to deal with non-monotone nonlinearities like, for example, $f(t, x, u)= \pm(\sin x) u^{2 k}+h(t, x), f(t, x, u)= \pm u^{2 k}+u^{2 k+1}+h(t, x)$.

Theorem 2. Let $f(t, x, u)=g(t, x, u)+h(t, x), h(t, x) \in N^{\perp}$ and

$$
g(t, x, u):=\beta(x) u^{2 k}+\mathcal{R}(t, x, u)
$$

where $\mathcal{R}, \partial_{t} \mathcal{R}, \partial_{u} \mathcal{R} \in C(\bar{\Omega} \times \mathbb{R}, \mathbb{R})$ satisfy ${ }^{2}$

$$
\|\mathcal{R}(\cdot, u)\|_{C(\bar{\Omega})}=\mathrm{o}\left(u^{2 k}\right), \quad\left\|\partial_{t} \mathcal{R}(\cdot, u)\right\|_{C(\bar{\Omega})}=\mathrm{O}\left(u^{2 k}\right), \quad\left\|\partial_{u} \mathcal{R}(\cdot, u)\right\|_{C(\bar{\Omega})}=\mathrm{o}\left(u^{2 k-1}\right),
$$

and $\beta \in C([0, \pi], \mathbb{R})$ verifies, for $x \in(0, \pi), \beta(x)>0($ or $\beta(x)<0)$ and $\beta(\pi-x)=\beta(x)$.

(i) (Existence) Assume there exists a weak solution $H \in E$ of $\square H=h$ such that

$$
H(t, x)>0 \quad(\text { or } H(t, x)<0) \quad \forall(t, x) \in \Omega .
$$

Then, for $\varepsilon$ small enough, there exists at least one weak solution $u \in E$ of (1.1)-(1.3) satisfying $\|u\|_{E} \leqslant C|\varepsilon|$.

(ii) (Regularity) If, moreover, $h \in H^{j}(\Omega) \cap C^{j-1}(\bar{\Omega}), \beta \in H^{j}((0, \pi)), \mathcal{R}, \partial_{t} \mathcal{R}, \partial_{u} \mathcal{R} \in C^{j}(\bar{\Omega} \times \mathbb{R}), j \geqslant 1$, then $u \in H^{j+1}(\Omega) \cap C_{0}^{j}(\bar{\Omega})$ and, for $j \geqslant 2$, $u$ is a classical solution.

Note that Theorem 2 does not require any growth condition on $g$ at infinity. In particular it applies for any analytic function $g(u)$ satisfying $g(0)=g^{\prime}(0)=\cdots=g^{2 k-1}(0)=0$ and $g^{2 k}(0) \neq 0$.

We now collect some comments on the previous results.

Remark 1.1. The assumption $h \in N^{\perp}$ is not of technical nature both in Theorem 1 and in Theorem 2 (at least if $g=g(x, u)=g(x,-u)=g(\pi-x, u))$. Indeed, if $h \notin N^{\perp}$, periodic solutions of problem (1.1)-(1.3) do not exist in any fixed ball $\left\{\|u\|_{L^{\infty}} \leqslant R\right\}, R>0$, for $\varepsilon$ small; see Remark 4.5.

We also note that the vector space of the $h \in L^{2}(\Omega)$ verifying $h(t, x)=h(t+\pi, \pi-x)$, introduced in [11], is a subspace of $N^{\perp}$.

Remark 1.2. In Theorem 2 hypotheses (1.8) and $\beta>0$ (or $\beta<0$ ) are assumed to prove the existence of a minimum of the "reduced action functional" $\Phi$, see (1.16). A sufficient condition implying (1.8) is $h>0$ a.e. in $\Omega$, see the "maximum principle" Proposition 4.11. This is also the key step to derive Theorem 1 from Theorem 2.

We also note that hypotheses (1.8) can be weakened, see Remark 4.4.

Remark 1.3 ((Regularity)). It is quite surprising that the weak solution $u$ of Theorems 1,2 is actually smooth. Indeed, while regularity always holds true for strictly monotone nonlinearities (see $[10,18]$ ), yet for weakly monotone $f$ it is not proved in general, unless the weak solution $u$ verifies $\left\|\Pi_{N} u\right\|_{L^{2}} \geqslant C>0$ (see [19]). Note, on the contrary, that the weak solution $u$ of Theorem 2 satisfies $\left\|\Pi_{N} u\right\|_{L^{2}}=\mathrm{O}(\varepsilon)$.

Moreover, assuming

$$
\left\|\partial_{t}^{l} \partial_{x}^{m} \partial_{u}^{n} \mathcal{R}\right\|_{C(\bar{\Omega})}=\mathrm{O}\left(u^{2 k-n}\right), \quad \forall 0 \leqslant l, n \leqslant j+1,0 \leqslant m \leqslant j, l+m+n \leqslant j+1
$$

we can also prove the estimate (see Remark 4.10)

$$
\|u\|_{H^{j+1}(\Omega)}+\|u\|_{C^{j}(\bar{\Omega})} \leqslant C|\varepsilon| .
$$

Remark 1.4 ((Multiplicity)). For non-monotone nonlinearities $f$ one can NOT in general expect uniqueness of the solutions. Actually, for $f(t, x, u)=g(x, u)+h(t, x)$ with $g(x, u)=g(x,-u), g(\pi-x, u)=g(x, u)$, there exist infinitely many $h \in N^{\perp}$ for which problem (1.1)-(1.3) has (at least) 3 solutions, see Remark 4.6.

Remark 1.5 ((Minimal period $))$. If $h(t, x)$ has minimal period $2 \pi$ w.r.t. time, then also the solution $u(t, x)$ has minimal period $2 \pi$, see Remark 4.7 .

\footnotetext{
2 The notation $f(z)=\mathrm{o}\left(z^{p}\right), p \in \mathbb{N}$, means that $f(z) /|z|^{p} \rightarrow 0$ as $z \rightarrow 0 . f(z)=\mathrm{O}\left(z^{p}\right)$ means that there exists a constant $C>0$ such that $|f(z)| \leqslant C|z|^{p}$ for all $z$ in a neighborhood of 0 .
} 
Finally, we extend the result of [18] proving existence of periodic solutions for non-monotone nonlinearities $f(t, x, u)$ obtained adding to a nonlinearity $\tilde{f}(t, x, u)$ as in [18] (i.e. $\left.\partial_{u} \tilde{f} \geqslant \beta>0\right)$ any non-monotone term $a(x, u)$ satisfying

$$
a(x,-u)=a(x, u), \quad a(\pi-x, u)=a(x, u)
$$

or

$$
a(x,-u)=-a(x, u), \quad a(\pi-x, u)=-a(x, u) .
$$

A prototype nonlinearity is $f(t, x, u)= \pm u^{2 k}+\tilde{f}(t, x, u)$ with $\partial_{u} \tilde{f} \geqslant \beta>0$.

Theorem 3. Let $f(t, x, u)=\tilde{f}(t, x, u)+a(x, u)$ where $f, \partial_{t} f, \partial_{u} f$ are continuous, $\partial_{u} \tilde{f} \geqslant \beta>0$ and $a(x, u)$ satisfy (1.11) or (1.12). Then, for $\varepsilon$ small enough, (1.1)-(1.3) has at least one weak solution $u \in E$. If moreover $f, \partial_{t} f$, $\partial_{u} f \in C^{j}(\bar{\Omega} \times \mathbb{R}), j \geqslant 1$, then $u \in H^{j+1}(\Omega) \cap C_{0}^{j}(\bar{\Omega})$.

In the next subsection we describe our method of proof.

\subsection{Scheme of the proof}

In order to find critical points of the Lagrangian action functional $\Psi: E \rightarrow \mathbb{R}$ defined in (1.5) we perform a variational Lyapunov-Schmidt reduction, decomposing the space $E:=H^{1}(\Omega) \cap C_{0}^{1 / 2}(\bar{\Omega})$ as

$$
E=V \oplus W
$$

where

$$
V:=N \cap H^{1}(\Omega) \quad \text { and } \quad W:=N^{\perp} \cap H^{1}(\Omega) \cap C_{0}^{1 / 2}(\bar{\Omega}) .
$$

Setting $u=v+w$ with $v \in V, w \in W$ and denoting by $\Pi_{N}$ and $\Pi_{N^{\perp}}$ the projectors from $L^{2}(\Omega)$ onto $N$ and $N^{\perp}$ respectively, problem (1.1)-(1.3) is equivalent to solve the kernel equation

$$
\Pi_{N} f(v+w, \varepsilon)=0
$$

and the range equation

$$
w=\varepsilon \square^{-1} \Pi_{N^{\perp}} f(v+w, \varepsilon)
$$

where $\square^{-1}: N^{\perp} \rightarrow N^{\perp}$ is the inverse of $\square$ and $f(u, \varepsilon)$ denotes the Nemitski operator associated to $f$, namely

$$
[f(u, \varepsilon)](t, x):=f(t, x, u, \varepsilon) .
$$

Remark 1.6. The usual approach (see $[12,18-20]$ ) is to find, first, by the monotonicity of $f$, the unique solution $v=v(w)$ of the kernel equation (1.13) and, next, to solve the range equation (1.14). On the other hand, for nonmonotone forcing terms, one can not in general solve uniquely the kernel equation - recall by Remark 1.4 that in general uniqueness of solutions does not hold. Therefore we must solve first the range equation and thereafter the kernel equation. For other applications of this approach to perturbation problems in critical point theory, see e.g. the forthcoming monograph by Ambrosetti and Malchiodi [1].

We solve, first, the range equation by means of a quantitative version of the Implicit Function Theorem, finding a solution $w:=w(v, \varepsilon) \in W$ of (1.14) with $\|w(v, \varepsilon)\|_{E}=\mathrm{O}(\varepsilon)$, see Proposition 3.2. Here no serious difficulties arise since $\square^{-1}$ acting on $W$ is a compact operator, due to the assumption $T=2 \pi$, see (2.2).

Remark 1.7. More in general, $\square^{-1}$ is compact on the orthogonal complement of $\operatorname{ker}(\square)$ whenever $T$ is a rational multiple of $2 \pi$. On the contrary, if $T$ is an irrational multiple of $2 \pi$, then $\square^{-1}$ is, in general, unbounded (a "small divisor" problem appears), but the kernel of $\square$ reduces to 0 (there is no bifurcation equation). For existence of periodic solutions in the case $T / 2 \pi$ is irrational see [17]. 
Once the range equation (1.14) has been solved by $w(v, \varepsilon) \in W$ it remains the infinite dimensional kernel equation (also called bifurcation equation)

$$
\Pi_{N} f(v+w(v, \varepsilon), \varepsilon)=0 .
$$

We note (see Lemma 3.3) that (1.15) is the Euler-Lagrange equation of the reduced Lagrangian action functional

$$
\Phi: V \longrightarrow \mathbb{R}, \quad \Phi(v):=\Phi(v, \varepsilon):=\Psi(v+w(v, \varepsilon), \varepsilon) .
$$

$\Phi$ lacks compactness properties and to find critical points of $\Phi$ we cannot rely on critical point theory.

Remark 1.8. Implementing an analogue Lyapunov-Schmidt reduction in the autonomous case (see [5]) it turns out that, in the corresponding reduced Lagrangian action functional, a further term proportional to $\|v\|_{H^{1}}^{2}$ is present. Therefore it is possible to apply critical point theory (e.g. the Mountain Pass Theorem) to find existence and multiplicity of solutions, see [6]. The elliptic term $\|v\|_{H^{1}}^{2}$ helps also in proving regularity results for the solutions.

We attempt to minimize $\Phi$.

We do not try to apply the direct methods of the calculus of variations. Indeed $\Phi$, even though it could possess some coercivity property, will not be convex (being $f$ non-monotone). Moreover, without assuming any growth condition on the nonlinearity $f$, the functional $\Phi$ could neither be well defined on any $L^{p}$-space.

Therefore we minimize $\Phi$ in any $\overline{B_{R}}:=\left\{v \in V,\|v\|_{H^{1}} \leqslant R\right\}, \forall R>0$, as in [18]. By standard compactness arguments $\Phi$ attains minimum at, say, $\bar{v} \in \overline{B_{R}}$. Since $\bar{v}$ could belong to the boundary $\partial \overline{B_{R}}, \bar{v}$ could not be a solution of (1.15) and we can only conclude the variational inequality

$$
D_{v} \Phi(\bar{v})[\varphi]=\int_{\Omega} f(\bar{v}+w(\bar{v}, \varepsilon), \varepsilon) \varphi \leqslant 0
$$

for any admissible variation $\varphi \in V$, i.e. if $\bar{v}+\theta \varphi \in \overline{B_{R}}, \forall \theta<0$ sufficiently small.

The heart of the existence proof of the weak solution $u$ of Theorems 1,2 and 3 is to obtain, choosing suitable admissible variations like in [18], the a-priori estimate $\|\bar{v}\|_{H^{1}}<R$ for some $R>0$, i.e. to show that $\bar{v}$ is an inner minimum point of $\Phi$ in $B_{R}$.

The strong monotonicity assumption $\left(\partial_{u} f\right)(t, x, u) \geqslant \beta>0$ would allow here to get such a-priori estimates by arguments similar to [18]. On the contrary, the main difficulty for proving Theorems 1, 2 and 3 which deal with non-monotone nonlinearities is to obtain such a-priori-estimates for $\bar{v}$.

The most difficult cases are the proof of Theorems 1 and 2. To understand the problem, let consider the particular nonlinearity $f(t, x, u)=u^{2 k}+h(t, x)$ of Theorem 1 . The even term $u^{2 k}$ does not give any contribution into the variational inequality (1.17) at the 0th-order in $\varepsilon$, since the right-hand side of (1.17) reduces, for $\varepsilon=0$, to

$$
\int_{\Omega}\left(\bar{v}^{2 k}+h(t, x)\right) \varphi=0, \quad \forall \varphi \in V
$$

by (2.18) and $h \in N^{\perp}$.

Therefore, for deriving, if ever possible, the required a-priori estimates, we have to develop the variational inequality (1.17) at higher orders in $\varepsilon$. We obtain

$$
0 \geqslant \int_{\Omega} 2 k \bar{v}^{2 k-1} \varphi w(\varepsilon, \bar{v})+\mathrm{O}\left(w^{2}(\varepsilon, \bar{v})\right)=\int_{\Omega} \varepsilon 2 k \bar{v}^{2 k-1} \varphi \square^{-1}\left(h+\bar{v}^{2 k}\right)+\mathrm{O}\left(\varepsilon^{2}\right)
$$

because $w(\bar{v}, \varepsilon)=\varepsilon \square^{-1}\left(\bar{v}^{2 k}+h\right)+\mathrm{o}(\varepsilon)$ (recall that $\left.\bar{v}^{2 k}, h \in N^{\perp}\right)$.

We now sketch how the $\varepsilon$-order term in the variational inequality (1.18) allows to prove an $L^{2 k}$-estimate for $\bar{v}$. Inserting the admissible variation $\varphi:=\bar{v}$ in (1.18) we get

$$
\int_{\Omega} H \bar{v}^{2 k}+\bar{v}^{2 k} \square^{-1} \bar{v}^{2 k} \leqslant \mathrm{O}(\varepsilon)
$$


where $H$ is a weak solution of $\square H=h$ which verifies $H(t, x)>0$ in $\Omega$ ( $H$ exists by the "maximum principle" Proposition 4.11). The crucial fact is that the first term in (1.19) satisfies the coercivity inequality

$$
\int_{\Omega} H v^{2 k} \geqslant c(H) \int_{\Omega} v^{2 k}, \quad \forall v \in V
$$

for some constant $c(H)>0$, see Proposition 4.2. The second term $\int_{\Omega} \bar{v}^{2 k} \square^{-1} \bar{v}^{2 k}$ will be negligible, $\varepsilon$-close to the origin, with respect to $\int_{\Omega} H v^{2 k}$ and (1.19), (1.20) will provide the $L^{2 k}$-estimate for $\bar{v}$.

We remark that the inequality $(1.20)$ is not trivial because $H$ vanishes at the boundary $(H(t, 0)=H(t, \pi)=0)$. Actually, the proof of (1.20) relies on the form $v(t, x)=\hat{v}(t+x)-\hat{v}(t-x)$ of the functions of $V$.

Next, we can obtain, choosing further admissible variations $\varphi$ in (1.18) and using inequalities similar to (1.20), an $L^{\infty}$-estimate for $\bar{v}$ and, finally, the required $H^{1}$-estimate, proving the existence of a weak solution $u \in E$, see Section 4.

Moreover, using similar techniques inspired to regularity theory and further suitable variations, we can also obtain a-priori estimates for the $L^{\infty}$-norm of the higher order derivatives of $\bar{v}$ and for its $H^{j}$-Sobolev norms. In this way we can prove the regularity of the solution $u$-fact quite surprising for non-monotone nonlinearities -, see Subsection 4.5.

Theorem 2 is proved developing such ideas and a careful analysis of the further term $\mathcal{R}$.

The proof of Theorem 3 is easier than for Theorems 1 and 2. Indeed the additional term $a(x, u)$ does not contribute into the variational inequality (1.17) at the 0 -order in $\varepsilon$, because $\int_{\Omega} a(x, \bar{v}) \varphi \equiv 0, \forall \varphi \in V$, by (2.19). Therefore the dominant term in the variational inequality (1.17) is provided by the monotone forcing term $\tilde{f}$ and the required a-priori estimates are obtained with arguments similar to [18], see Section 5.

Notations. $\Omega:=\mathbb{T} \times(0, \pi)$ where $\mathbb{T}:=\mathbb{R} / 2 \pi \mathbb{Z}$. We denote by $C^{j}(\bar{\Omega})$ the Banach space of functions $u: \bar{\Omega} \rightarrow \mathbb{R}$ with $j$ derivatives in $\Omega$ continuous up to the boundary $\partial \Omega$, endowed with the standard norm $\|\cdot\|_{C^{j}} \cdot C_{0}^{j}(\bar{\Omega}):=$ $C^{j}(\bar{\Omega}) \cap C_{0}(\bar{\Omega})$ where $C_{0}(\bar{\Omega})$ is the space of real valued continuous functions satisfying $u(t, 0)=u(t, \pi)=0$. Moreover $H^{j}(\Omega):=W^{j, 2}(\Omega)$ are the usual Sobolev spaces with scalar product $\langle\cdot, \cdot\rangle_{H^{j}}$ and norm \|\|$_{H^{j}(\Omega)}^{2}$. Here $C^{j}(\mathbb{T})$ denotes the Banach space of periodic functions $u: \mathbb{T} \rightarrow \mathbb{R}$ with $j$ continuous derivatives. Finally, $H^{j}(\mathbb{T})$ is the usual Sobolev space of $2 \pi$-periodic functions.

\section{Preliminaries}

We first collect some important properties on the D'Alembertian operator $\square$.

Definition 2.1. Given $f(t, x) \in L^{2}(\Omega)$, a function $u \in L^{2}(\Omega)$ is said to be a weak solution of $\square u=f$ in $\Omega$ satisfying the boundary conditions $u(t, 0)=u(t, \pi)=0$, iff

$$
\int_{\Omega} u \square \varphi=\int_{\Omega} f \varphi \quad \forall \varphi \in C_{0}^{2}(\bar{\Omega}) .
$$

It is easily verified that, if $u \in C^{2}(\bar{\Omega})$ is a weak solution of $\square u=f$ according to Definition 2.1, then $u$ is a classical solution and $u(t, 0)=u(t, \pi)=0$.

The kernel $N \subset L^{2}(\Omega)$ of the D'Alembertian operator $\square$, i.e. the space of weak solutions of the homogeneous linear equation $\square v=0$ verifying the Dirichlet boundary conditions $v(t, 0)=v(t, \pi)=0$, is the subspace $N$ defined in (1.6). $N$ coincides with the closure in $L^{2}(\Omega)$ of the classical solution of $\square v=0$ verifying Dirichlet boundary conditions which, as well known, are of the form $v(t, x)=\hat{v}(t+x)-\hat{v}(t-x), \hat{v} \in C^{2}(\mathbb{T})$.

Using Fourier series we can also characterize $N$ as

$$
N=\left\{v(t, x) \in L^{2}(\Omega) \mid v(t, x)=\sum_{j \in \mathbb{Z}} a_{j} \mathrm{e}^{\mathrm{i} j t} \sin j x \text { with } \sum_{j \in \mathbb{Z}}\left|a_{j}\right|^{2}<\infty\right\} .
$$

The range of $\square$ in $L^{2}(\Omega)$ is 


$$
\begin{aligned}
N^{\perp} & :=\left\{f \in L^{2}(\Omega) \mid \int_{\Omega} f v=0, \forall v \in N\right\} \\
& =\left\{f(t, x) \in L^{2}(\Omega) \mid f(t, x)=\sum_{\substack{l \in \mathbb{Z}, j \geqslant 1 \\
j \neq|l|}} f_{l j} \mathrm{e}^{\mathrm{i} l t} \sin j x \text { with } \sum_{\substack{l \in \mathbb{Z}, j \geqslant 1 \\
j \neq|l|}}\left|f_{l j}\right|^{2}<\infty\right\},
\end{aligned}
$$

i.e. $\forall f(t, x) \in N^{\perp}$ there exists a unique weak solution $u=\square^{-1} f \in N^{\perp}$ of $\square u=f$.

Furthermore $\square^{-1}$ is a bounded operator such that

$$
\square^{-1}: N^{\perp} \longrightarrow N^{\perp} \cap H^{1}(\Omega) \cap C_{0}^{1 / 2}(\bar{\Omega}),
$$

i.e. there exists a suitable constant $\underline{c} \geqslant 1$ such that

$$
\left\|\square^{-1} f\right\|_{E} \leqslant \underline{c}\|f\|_{L^{2}} \quad \forall f \in L^{2}(\Omega)
$$

where $\|u\|_{E}:=\|u\|_{H^{1}}+\|u\|_{C^{1 / 2}}$. By (2.2) and the compact embedding $H^{1}(\Omega) \hookrightarrow L^{2}(\Omega)$, the operator $\square^{-1}: N^{\perp} \rightarrow$ $N^{\perp}$ is compact.

These assertions follow easily from the Fourier series representation (see e.g. [10])

$$
f(t, x):=\sum_{j \geqslant 1, j \neq|l|} f_{l j} \mathrm{e}^{\mathrm{i} l t} \sin j x \Rightarrow u=\square^{-1} f:=\sum_{j \geqslant 1, j \neq|l|} \frac{f_{l j}}{-l^{2}+j^{2}} \mathrm{e}^{\mathrm{i} l t} \sin j x
$$

noting that $u$ is a weak solution of (1.1) (according to Definition 2.1) iff $u_{l j}=f_{l j} /\left(-l^{2}+j^{2}\right) \forall l \in \mathbf{Z}, j \geqslant 1$, see e.g. $[12,15]$.

To continue, $\square^{-1}$ is a bounded operator also between the spaces

$$
L^{\infty}(\Omega) \longrightarrow C^{0,1}(\bar{\Omega}), \quad H^{k}(\Omega) \longrightarrow H^{k+1}(\Omega), \quad C^{k}(\bar{\Omega}) \longrightarrow C^{k+1}(\bar{\Omega})
$$

as follows by the integral formula for $u=\square^{-1} f=\Pi_{N^{\perp}} \psi$ where (see e.g. [9,16])

$$
\psi(t, x):=-\frac{1}{2} \int_{x}^{\pi} \int_{t+x-\xi}^{t-x+\xi} f(\xi, \tau) \mathrm{d} \tau \mathrm{d} \xi+c \frac{\pi-x}{\pi},
$$

$\Pi_{N^{\perp}}: L^{2}(\Omega) \rightarrow N^{\perp}$ is the orthogonal projector onto $N^{\perp}$ and

$$
c:=\frac{1}{2} \int_{0}^{\pi} \int_{t-\xi}^{t+\xi} f(\xi, \tau) \mathrm{d} \tau \mathrm{d} \xi \equiv \frac{1}{2} \int_{0}^{\pi} \int_{-\xi}^{\xi} f(\xi, \tau) \mathrm{d} \tau \mathrm{d} \xi \equiv \text { const }
$$

is a constant independent of $t$, because ${ }^{3} f \in N^{\perp}$.

$\overline{3}$ We have that $2 c=\int_{\mathcal{T}(t)} f=\lim _{n \rightarrow \infty} \int_{\mathcal{T}(t)} f_{n}$ where $\mathcal{T}(t):=\{(\tau, \xi) \in \Omega$ s.t. $t-\xi<\tau<t+\xi, 0<\xi<\pi\}$ and

$$
f_{n}(t, x):=\sum_{\substack{|l|, j \leqslant n \\ j \neq|l|}} f_{l, j} \mathrm{e}^{\mathrm{i} l t} \sin j x \stackrel{L^{2}}{\rightarrow} \sum_{j \neq|l|} f_{l, j} \mathrm{e}^{\mathrm{i} l t} \sin j x=f(t, x) \in N^{\perp} .
$$

The claim follows since $\int_{\mathcal{T}(t)} f_{n}$ is, for any $n$, independent on $t$ :

$$
\begin{aligned}
\int_{\mathcal{T}(t)} f_{n} & =\int_{0}^{\pi} \sum_{\substack{l l|, j \leqslant n \\
j \neq| l \mid}} f_{l, j} \sin j \xi \int_{t-\xi}^{t+\xi} \mathrm{e}^{\mathrm{i} l t} \mathrm{~d} \tau \mathrm{d} \xi \\
& =\sum_{1 \leqslant j \leqslant n} f_{0, j} \int_{0}^{\pi} 2 \xi \sin j \xi \mathrm{d} \xi+\sum_{\substack{|l|, j \leqslant n \\
j \neq|l|, l \neq 0}} 2 \frac{f_{l, j}}{l} \mathrm{e}^{\mathrm{i} l t} \int_{0}^{\pi} \sin j \xi \sin l \xi \mathrm{d} \xi=\sum_{1 \leqslant j \leqslant n} f_{0, j} \int_{0}^{\pi} 2 \xi \sin j \xi \mathrm{d} \xi .
\end{aligned}
$$


We also have, since $\partial_{t}^{j} H$ is a weak solution of $\square\left(\partial_{t}^{j} H\right)=\partial_{t}^{j} h$ and (2.1) applies,

$$
h \in H^{j}(\Omega) \quad \Longrightarrow \quad \partial_{t}^{j} H \in C_{0}^{1 / 2}(\bar{\Omega}) \text {. }
$$

Finally, the projector $\Pi_{N}: L^{2}(\Omega) \rightarrow N$ can be written as $\Pi_{N} u=p(t+x)-p(t-x)$ where

$$
p(y):=\frac{1}{2 \pi} \int_{0}^{\pi}[u(y-s, s)-u(y+s, s)] \mathrm{d} s
$$

and therefore, since $u \in C^{j}(\bar{\Omega}) \Rightarrow p \in C^{j}(\mathbb{T})$ and $u \in H^{j}(\Omega) \Rightarrow p \in H^{j}(\mathbb{T})$,

$$
\begin{array}{ll}
\Pi_{N}, \Pi_{N \perp}: C^{j}(\bar{\Omega}) \rightarrow C^{j}(\bar{\Omega}) & \text { are bounded, } \\
\Pi_{N}, \Pi_{N \perp}: H^{j}(\Omega) \rightarrow H^{j}(\Omega) & \text { are bounded. }
\end{array}
$$

\subsection{Kernel properties and technical lemmata}

Let define, for $0 \leqslant \alpha<1 / 2$,

$$
\Omega_{\alpha}:=\mathbb{T} \times(\alpha \pi, \pi-\alpha \pi) \subseteq \Omega .
$$

Lemma 2.2. Let $a \in L^{1}(\Omega)$.

$$
\int_{\Omega_{\alpha}} a(t, x) \mathrm{d} t \mathrm{~d} x=\frac{1}{2} \int_{0}^{2 \pi} \int_{-2 \pi+s_{+}+2 \alpha \pi}^{s_{+}-2 \alpha \pi} a\left(\frac{s_{+}+s_{-}}{2}, \frac{s_{+}-s_{-}}{2}\right) \mathrm{d} s_{-} \mathrm{d} s_{+} .
$$

In particular for $p, q \in L^{1}(\mathbb{T})$

$$
\int_{\Omega_{\alpha}} p(t+x) q(t-x) \mathrm{d} t \mathrm{~d} x=\frac{1}{2} \int_{0}^{2 \pi} p(s) \mathrm{d} s \int_{0}^{2 \pi} q(s) \mathrm{d} s-\frac{1}{2} \int_{-2 \alpha \pi}^{2 \alpha \pi} \int_{0}^{2 \pi} p(y) q(z+y) \mathrm{d} y \mathrm{~d} z
$$

and

$$
\int_{\Omega_{\alpha}} p(t+x) \mathrm{d} t \mathrm{~d} x=\int_{\Omega_{\alpha}} p(t-x) \mathrm{d} t \mathrm{~d} x=\pi(1-2 \alpha) \int_{0}^{2 \pi} p(s) \mathrm{d} s .
$$

Moreover, given $f, g: \mathbb{R} \rightarrow \mathbb{R}$ continuous,

$$
\int_{\Omega_{\alpha}} f(p(t+x)) g(p(t-x)) \mathrm{d} t \mathrm{~d} x=\int_{\Omega_{\alpha}} f(p(t-x)) g(p(t+x)) \mathrm{d} t \mathrm{~d} x .
$$

Proof. In Appendix A.

Lemma 2.3. For any $v=\hat{v}(t+x)-\hat{v}(t-x):=v_{+}-v_{-} \in N$

$$
\|v\|_{L^{2}(\Omega)}^{2}=2 \pi\|\hat{v}\|_{L^{2}(\mathbb{T})}^{2}=2 \pi \int_{0}^{2 \pi} \hat{v}^{2}
$$

\section{Moreover}

$$
\begin{aligned}
& \left\|v_{t}\right\|_{L^{2}(\Omega)}^{2}=\left\|v_{x}\right\|_{L^{2}(\Omega)}^{2}=2 \pi\left\|\hat{v}^{\prime}\right\|_{L^{2}(\mathbb{T})}^{2} \quad \forall v \in N \cap H^{1}(\Omega), \\
& \|\hat{v}\|_{L^{\infty}(\mathbb{T})} \leqslant\|v\|_{L^{\infty}(\Omega)} \leqslant 2\|\hat{v}\|_{L^{\infty}(\mathbb{T})} \quad \forall v \in N \cap L^{\infty}(\Omega), \\
& \|v\|_{L^{\infty}(\Omega)} \leqslant\|v\|_{H^{1}(\Omega)} \quad \forall v \in N \cap H^{1}(\Omega) .
\end{aligned}
$$


Proof. In Appendix A.

Lemma 2.4. Let $\varphi_{1}, \ldots, \varphi_{2 k+1} \in N$ and $\varphi_{1} \cdots \varphi_{2 k+1} \in L^{1}(\Omega)$. Then

$$
\int_{\Omega_{\alpha}} \varphi_{1} \cdots \varphi_{2 k+1}=0 .
$$

In particular $\varphi_{1} \cdots \varphi_{2 k} \in N^{\perp}$.

Moreover, if $a: \Omega \rightarrow \mathbb{R}$ satisfies (i) $a(x, u)=a(\pi-x, u)$ and $a(x, u)=a(x,-u)$ or (ii) $a(x, u)=-a(\pi-x, u)$ and $a(x, u)=-a(x,-u)$, then

$$
\int_{\Omega_{\alpha}} a(x, v) \varphi=0 \quad \forall v \in N \cap L^{\infty}, \varphi \in N
$$

and

$$
\int_{\Omega_{\alpha}}\left(\partial_{u} a\right)(x, v) \varphi_{1} \varphi_{2}=0 \quad \forall v \in N \cap L^{\infty}, \varphi_{1}, \varphi_{2} \in N .
$$

Proof. In Appendix A.

Lemma 2.5. The following inequalities hold:

$$
\begin{aligned}
& (a-b)^{2 k} \leqslant 2^{2 k-1}\left(a^{2 k}+b^{2 k}\right) \quad \forall a, b \in \mathbb{R}, \\
& (a-b)^{2 k} \geqslant a^{2 k}+b^{2 k}-2 k\left(a^{2 k-1} b+a b^{2 k-1}\right) \quad \forall a, b \in \mathbb{R}, k \in \mathbb{N}, k \geqslant 2, \\
& (a+b)^{2 k-1}-a^{2 k-1} \geqslant 4^{1-k} b^{2 k-1} \quad \forall a \in \mathbb{R}, b>0, k \in \mathbb{N}^{+} .
\end{aligned}
$$

Proof. In Appendix A.

\subsection{Generalities about the difference quotients}

For $f \in L^{2}(\Omega)$ we define the difference quotient of size $h \in \mathbb{R} \backslash\{0\}$

$$
\left(D_{h} f\right)(t, x):=\frac{f(t+h, x)-f(t, x)}{h}
$$

and the $h$-translation

$$
\left(T_{h} f\right)(t, x):=f(t+h, x)
$$

with respect to time.

The following lemma collects some elementary properties of the difference quotient.

Lemma 2.6. Let $f, g \in L^{2}(\Omega), h \in \mathbb{R} \backslash\{0\}$. The following holds

(i) Leibnitz rule:

$$
\begin{aligned}
& D_{h}(f g)=\left(D_{h} f\right) g+T_{h} f D_{h} g, \\
& D_{h} f^{m}=\left(D_{h} f\right) \sum_{j=0}^{m-1} f^{m-j-1} T_{h} f^{j}=m\left(D_{h} f\right) f^{m-1}+\left(D_{h} f\right) \sum_{j=0}^{m-1} f^{m-j-1}\left(T_{h} f^{j}-f^{j}\right), \\
& \int_{\Omega} D_{h}(f g)=\int_{\Omega}\left(D_{h} f\right) g+f\left(D_{-h} g\right) ;
\end{aligned}
$$


(ii) integration by parts:

$$
\int_{\Omega} f\left(D_{-h} g\right)=-\int_{\Omega}\left(D_{h} f\right) g ;
$$

(iii) weak derivative: If there exists a constant $C>0$ such that $\forall$ small

$$
\left\|D_{h} f\right\|_{L^{2}} \leqslant C \Rightarrow \text { then } f \text { has a weak derivative } f_{t} \text { and }\left\|f_{t}\right\|_{L^{2}} \leqslant C \text {. }
$$

Moreover, if $f$ has a weak derivative $f_{t} \in L^{2}(\Omega)$, then

(iv) estimate on the difference quotient:

$$
\left\|D_{h} f\right\|_{L^{2}(\Omega)} \leqslant\left\|f_{t}\right\|_{L^{2}(\Omega)} ;
$$

(v) convergence:

$$
D_{h} f \stackrel{L^{2}}{\longrightarrow} f_{t} \text { as } h \longrightarrow 0 .
$$

Proof. In Appendix A.

\section{The Lyapunov-Schmidt decomposition}

\subsection{The range equation}

We first solve the range equation (1.14) applying the following quantitative version of the Implicit Function Theorem, whose standard proof is omitted.

Proposition 3.1. Let $X, Y, Z$ be Banach spaces and $x_{0} \in X, y_{0} \in Y$. Fix $r, \rho>0$ and define $X_{r}:=\{x \in X$ s.t. $\left.\left\|x-x_{0}\right\|_{X}<r\right\}$ and $Y_{\rho}:=\left\{y \in Y\right.$ s.t. $\left.\left\|y-y_{0}\right\|_{Y}<\rho\right\}$. Let $\mathcal{F} \in \mathcal{C}^{1}(U, Z)$ where $\overline{Y_{\rho}} \times \overline{X_{r}} \subset U \subset Y \times X$ is an open set. Suppose that

$$
\mathcal{F}\left(y_{0}, x_{0}\right)=0
$$

and that $D_{y} \mathcal{F}\left(y_{0}, x_{0}\right) \in \mathcal{L}(Y, Z)$ is invertible. Let $T:=\left(D_{y} \mathcal{F}\left(y_{0}, x_{0}\right)\right)^{-1}$ and $\|T\|:=\|T\|_{\mathcal{L}(Z, Y)}$ be its norm. If

$$
\begin{aligned}
& \sup _{\overline{X_{r}}}\left\|\mathcal{F}\left(y_{0}, x\right)\right\|_{Z} \leqslant \frac{\rho}{2\|T\|}, \\
& \sup _{\overline{Y_{\rho}} \times \overline{X_{r}}}\left\|\operatorname{Id}_{Y}-T D_{y} \mathcal{F}(y, x)\right\|_{\mathcal{L}(Y, Y)} \leqslant \frac{1}{2},
\end{aligned}
$$

then there exists $y \in \mathcal{C}^{1}\left(X_{r}, \overline{Y_{\rho}}\right)$ such that $\mathcal{F}(y(x), x) \equiv 0$.

Applying Proposition 3.1 to the range equation (1.14) we derive:

Proposition 3.2. Let $f=f(t, x, u, \varepsilon), f_{u}:=\partial_{u} f$ and $\varepsilon \partial_{\varepsilon} f$ be continuous on $\bar{\Omega} \times \mathbb{R} \times[-1,1]$. Then $\forall R>0$ there exists a unique function

$$
w=w(v, \varepsilon) \in \mathcal{C}^{1}\left(\left\{\|v\|_{L^{\infty}}<2 R\right\} \times\left\{|\varepsilon|<\varepsilon_{0}(R)\right\},\left\{\|w\|_{E} \leqslant C_{0}(R)|\varepsilon|\right\}\right)
$$

solving the range equation (1.14), where $\varepsilon_{0}(R):=1 / 2 C_{0}(R)$ and $^{4}$

$$
C_{0}(R):=1+\sqrt{2} \pi \underline{c}_{\bar{\Omega} \times\{|u| \leqslant 3 R+1\} \times\{|\varepsilon| \leqslant 1\}}\left[|f(t, x, u, \varepsilon)|+\left|f_{u}(t, x, u, \varepsilon)\right|\right] .
$$


Proof. Let $X:=V \times \mathbb{R}, Y=Z:=W$ (namely $x:=(v, \varepsilon)$ and $y:=w)$ and $\|x\|_{X}:=\|v\|_{L^{\infty}}+\frac{R}{\varepsilon_{0}(R)}|\varepsilon|$. Let also $x_{0}:=$ $(0,0), y_{0}:=0, r:=3 R, \rho:=1, \mathcal{F}(y, x):=\mathcal{F}(w, v, \varepsilon):=w-\varepsilon \square^{-1} \Pi_{N^{\perp}} f(v+w, \varepsilon)$ and $U:=W \times V \times(-1,1)$. Note that $\mathcal{F}(\cdot, \cdot) \in C^{1}$ since the Nemitski operator $\varepsilon f \in C^{1}\left(E \times(-1,1), L^{2}(\Omega)\right)$ and (2.1) holds. Moreover (3.1) holds and $T=\operatorname{Id}_{W}$ (hence $\|T\|=1$ ).

If $\|v\|_{L^{\infty}} \leqslant 3 R$ and $\|w\|_{L^{\infty}} \leqslant\|w\|_{E} \leqslant 1$, then

$$
|v(t, x)+w(t, x)| \leqslant 3 R+1, \quad \forall(t, x) \in \bar{\Omega} .
$$

Using (2.2), Bessel inequality $\left\|\Pi_{N^{\perp}} f\right\|_{L^{2}} \leqslant\|f\|_{L^{2}},\|f\|_{L^{2}(\Omega)} \leqslant \sqrt{2} \pi\|f\|_{L^{\infty}(\Omega)}$ and estimate (3.6), we obtain, $\forall|\varepsilon| \leqslant \varepsilon_{0}(R)$ and $\|v\|_{L^{\infty}} \leqslant 3 R$,

$$
\|\mathcal{F}(0, v, \varepsilon)\|_{E} \leqslant|\varepsilon| \underline{c}\|f(v, \varepsilon)\|_{L^{2}} \leqslant|\varepsilon| \sqrt{2} \pi \underline{c}\|f(v, \varepsilon)\|_{L^{\infty}} \leqslant C_{0}(R)|\varepsilon| \leqslant \frac{1}{2}
$$

where $C_{0}(R)$ is defined in (3.5). Hence (3.2) follows from (3.7).

Since

$$
D_{w} \mathcal{F}(w, v, \varepsilon)[\widetilde{w}]=\widetilde{w}-\varepsilon \square^{-1} \Pi_{N^{\perp}}\left(f_{u}(v+w, \varepsilon) \widetilde{w}\right) \quad \forall \widetilde{w} \in W,
$$

we deduce, arguing as before, $\forall\|v\|_{L^{\infty}} \leqslant 3 R, \forall\|w\|_{E} \leqslant 1, \forall|\varepsilon| \leqslant \varepsilon_{0}(R)$,

$$
\sup _{\|\widetilde{w}\|_{E}=1}\left\|\widetilde{w}-D_{w} \mathcal{F}(w, v, \varepsilon)[\widetilde{w}]\right\|_{E} \leqslant|\varepsilon| \sqrt{2} \pi \underline{c}\left\|f_{u}(v+w, \varepsilon)\right\|_{L^{\infty}} \leqslant \varepsilon_{0}(R) C_{0}(R)=\frac{1}{2}
$$

and (3.3) follows. Now we can apply Proposition 3.1 finding a function $w=w(v, \varepsilon) \in C^{1}\left(\left\{\|x\|_{X}<r\right\}, \bar{W}_{1}\right)$ satisfying the range equation (1.14). Finally, note that

$$
\left\{\|v\|_{L^{\infty}}<2 R\right\} \times\left\{|\varepsilon|<\varepsilon_{0}(R)\right\} \subset\left\{\|x\|_{X}<r=3 R\right\}
$$

and, arguing as above,

$$
\|w(v, \varepsilon)\|_{E}=\left\|\varepsilon \square^{-1} \Pi_{N^{\perp}} f(v+w(v, \varepsilon) ; \varepsilon)\right\|_{E} \leqslant|\varepsilon| \sqrt{2} \pi \underline{c}\|f(v+w ; \varepsilon)\|_{L^{\infty}} \leqslant|\varepsilon| C_{0}(R),
$$

whence (3.4) follows.

\subsection{The kernel equation}

Once the range equation (1.14) has been solved by $w(v, \varepsilon) \in W$ there remains the infinite dimensional kernel equation (1.15).

Since $V$ is dense in $N$ with the $L^{2}$-norm, Eq. (1.15) is equivalent to

$$
\int_{\Omega} f(v+w(v, \varepsilon), \varepsilon) \varphi=0 \quad \forall \varphi \in V
$$

which is the Euler-Lagrange equation of the reduced Lagrangian action functional $\Phi: V \rightarrow \mathbb{R}, \Phi(v):=\Phi(v, \varepsilon):=$ $\Psi(v+w(v, \varepsilon), \varepsilon)$, defined in (1.16). Actually:

Lemma 3.3. $\Phi \in \mathcal{C}^{1}\left(\left\{\|v\|_{H^{1}}<2 R\right\}, \mathbb{R}\right)$ and a critical point $\bar{v}$ of $\Phi$ is a weak solution of the kernel equation (1.15).

Moreover $\Phi$ can be written as

$$
\Phi(v)=\varepsilon \int_{\Omega}\left[F(v+w(v) ; \varepsilon)-\frac{1}{2} f(v+w(v) ; \varepsilon) w(v)\right] \mathrm{d} t \mathrm{~d} x
$$

and

$$
\left\|v_{n}\right\|_{H^{1}},\|\bar{v}\|_{H^{1}} \leqslant R, v_{n} \stackrel{L^{\infty}}{\longrightarrow} \bar{v} \Longrightarrow \Phi\left(v_{n}\right) \longrightarrow \Phi(\bar{v}) .
$$

Proof. Since $\Psi(\cdot, \varepsilon) \in C^{1}(E, \mathbb{R})$ and, by Proposition 3.2, $w(\cdot, \varepsilon) \in C^{1}\left(\left\{\|v\|_{H^{1}}<2 R\right\}, \mathbb{R}\right)$ (note that $\left\{\|v\|_{H^{1}}<2 R\right\} \subset$ $\left\{\|v\|_{L^{\infty}}<2 R\right\}$ by $\left.(2.16)\right)$, then $\Phi \in \mathcal{C}^{1}\left(\left\{\|v\|_{H^{1}}<2 R\right\}, \mathbb{R}\right)$ and

$$
D \Phi(v)[\varphi]=D \Psi(v+w(v))[\varphi+d w(v)[\varphi]] \quad \forall \varphi \in V .
$$


We claim that, since $w=w(v) \in E$ is a weak solution of the range equation (1.14) and $\widetilde{w}:=d w(v)[\varphi] \in W$, then

$$
D \Psi(v+w(v))[d w(v)[\varphi]]=0 .
$$

Indeed, since $v_{t}, v_{x} \in N$ and $\widetilde{w}_{t}, \widetilde{w}_{x} \in N^{\perp}$,

$$
\begin{aligned}
D \Psi(v+w)[\widetilde{w}] & =\int_{\Omega}(v+w)_{t} \widetilde{w}_{t}-(v+w)_{x} \widetilde{w}_{x}+\varepsilon f(v+w, \varepsilon) \widetilde{w} \\
& =\int_{\Omega} w_{t} \widetilde{w}_{t}-w_{x} \widetilde{w}_{x}+\varepsilon \Pi_{N^{\perp}} f(v+w, \varepsilon) \widetilde{w}=0
\end{aligned}
$$

because $w \in E$ is a weak solution of the range equation $\square w=\varepsilon \Pi_{N^{\perp}} f(v+w, \varepsilon)$ and $w(t, 0)=w(t, \pi)=0$.

By (3.12), (3.13) and since $w_{t}, w_{x} \in N^{\perp}$ and $\varphi_{t}, \varphi_{x} \in N$

$$
\begin{aligned}
D \Phi(v)[\varphi] & =D \Psi(v+w)[\varphi]=\int_{\Omega}(v+w)_{t} \varphi_{t}-(v+w)_{x} \varphi_{x}+\varepsilon f(v+w, \varepsilon) \varphi \\
& =\int_{\Omega} v_{t} \varphi_{t}-v_{x} \varphi_{x}+\varepsilon f(v+w, \varepsilon) \varphi=\varepsilon \int_{\Omega} f(v+w, \varepsilon) \varphi=\varepsilon \int_{\Omega} \Pi_{N^{\perp}} f(v+w, \varepsilon) \varphi
\end{aligned}
$$

where in (3.15) we used $\int_{\Omega} v_{t} \varphi_{t}-v_{x} \varphi_{x}=0$ since $v, \varphi \in V$.

Now we prove (3.10) as in [5]. Since $v_{t}, v_{x} \in N, w_{t}, w_{x} \in N^{\perp}$ and (2.14)

$$
\Phi(v)=\int_{\Omega} \frac{(v+w(v))_{t}^{2}}{2}-\frac{(v+w(v))_{x}^{2}}{2}+\varepsilon F(v+w(v) ; \varepsilon)=\int_{\Omega} \frac{(w(v))_{t}^{2}}{2}-\frac{(w(v))_{x}^{2}}{2}+\varepsilon F(v+w(v) ; \varepsilon)
$$

and since $\int_{\Omega}(w(v))_{t}^{2}-(w(v))_{x}^{2}=-\int_{\Omega} \varepsilon f(v+w(v) ; \varepsilon) w(v)$ we deduce (3.10).

Finally let us prove (3.11). Setting $w_{n}:=w\left(v_{n}, \varepsilon\right)$ and $\bar{w}:=w(\bar{v}, \varepsilon)$, we have

$$
\begin{aligned}
\left|\int_{\Omega} F\left(v_{n}+w_{n}\right)-F(\bar{v}+\bar{w})\right| & \leqslant \max _{\bar{\Omega} \times\{|u| \leqslant R+1\} \times\{|\varepsilon| \leqslant 1\}}|f(t, x, u, \varepsilon)| \int_{\Omega}\left|v_{n}-\bar{v}+w_{n}-\bar{w}\right| \\
& \leqslant C_{0}(R)\left(\left\|v_{n}-\bar{v}\right\|_{L^{1}}+\left\|w_{n}-\bar{w}\right\|_{L^{1}}\right) \longrightarrow 0
\end{aligned}
$$

as $n \rightarrow \infty$, by the fact that $\left\|v_{n}-\bar{v}\right\|_{L^{\infty}} \rightarrow 0$ and (3.4). An analogous estimate holds for the second term in the integral in (3.10).

By standard compactness argument the functional $\Phi$ attains minimum (resp. maximum) in $\overline{B_{R}}:=\{v \in V$, $\left.\|v\|_{H^{1}} \leqslant R\right\}, \forall R>0$. Indeed, let $v_{n} \in \overline{B_{R}}$ be a minimizing (resp. maximizing) sequence $\Phi\left(v_{n}\right) \rightarrow \inf _{\overline{B_{R}}} \Phi$. Since $\left\{v_{n}\right\}_{n \in \mathbb{N}}$ is bounded in $N \cap H^{1}$, up to a subsequence $v_{n} \stackrel{H^{1}}{\rightarrow} \bar{v}$ for some $\bar{v}=\bar{v}(R, \varepsilon) \in \overline{B_{R}}$. Moreover by the compact embedding $H^{1}(\mathbb{T}) \hookrightarrow L^{\infty}(\mathbb{T})$ we can also assume $v_{n} \stackrel{L^{\infty}}{\longrightarrow} \bar{v}$ (since $\hat{v}_{n} \stackrel{L^{\infty}(\mathbb{T})}{\longrightarrow} \hat{\bar{v}}$ ) and therefore, by (3.11), $\bar{v}$ is a minimum (resp. maximum) point of $\Phi$ restricted to $\overline{B_{R}}$.

Since $\bar{v}$ could belong to the boundary $\partial \overline{B_{R}}$ we only have the variational inequality (1.17) for any admissible variation $\varphi \in V$, namely for any $\varphi \in V$ such that $\bar{v}+\theta \varphi \in \overline{B_{R}}, \forall \theta<0$ sufficiently small. As proved by Rabinowitz [18], a sufficient condition for $\varphi \in V$ to be an admissible variation is the positivity of the scalar product

$$
\langle\bar{v}, \varphi\rangle_{H^{1}}>0 \text {. }
$$

The heart of the existence proof of Theorems 1, 2 and 3 is to obtain, choosing suitable admissible variations, the a-priori estimate $\|\bar{v}\|_{H^{1}}<R$ for some $R>0$, i.e. to show that $\bar{v}$ is an inner minimum (resp. maximum) point of $\Phi$ in $B_{R}$.

\section{Proof of Theorems 1 and 2}

The main difficulty for proving Theorems 1 and 2 is to obtain the fore mentioned a-priori-estimate for $\bar{v}$. 


\subsection{Proof of Theorem 2}

As anticipated in Subsection 1.2, we look for small amplitude solutions of (1.1) with forcing term $f(t, x, u)=$ $g(t, x, u)+h(t, x)$ where $g(t, x, u)=\beta(x) u^{2 k}+\mathcal{R}(t, x, u), h(t, x) \in N^{\perp}$ and $\mathcal{R}(t, x, u)$ satisfies (1.7).

Perform the change of variables $u=\varepsilon(H+\tilde{u})$ and set $\tilde{\varepsilon}:=\varepsilon^{2 k}$

$$
\begin{aligned}
\square \tilde{u}=g(t, x, \varepsilon(H+\tilde{u})) & =\varepsilon^{2 k}\left[\beta(x)(H+\tilde{u})^{2 k}+\varepsilon^{-2 k} \mathcal{R}(t, x, \varepsilon(H+\tilde{u}))\right] \\
& =\tilde{\varepsilon}\left[\beta(x)(H+\tilde{u})^{2 k}+\tilde{\varepsilon}^{-1} \mathcal{R}\left(t, x, \tilde{\varepsilon}^{1 /(2 k)}(H+\tilde{u})\right)\right] .
\end{aligned}
$$

Recalling $\tilde{u} \rightarrow u, \tilde{\varepsilon} \rightarrow \varepsilon$, we look for solutions of the problem

$$
\left\{\begin{array}{l}
\square u=\varepsilon f(t, x, u ; \varepsilon), \\
u(t, 0)=u(t, \pi)=0, \\
u(t+2 \pi, x)=u(t, x)
\end{array}\right.
$$

where the nonlinear forcing term is

$$
f(t, x, u ; \varepsilon):=\beta(x)(H+u)^{2 k}+\mathcal{R}^{*}(t, x, u ; \varepsilon)
$$

and

$$
\mathcal{R}^{*}(t, x, u ; \varepsilon):=\varepsilon^{-1} \mathcal{R}\left(t, x, \varepsilon^{1 /(2 k)}(H(t, x)+u)\right) .
$$

Moreover, eventually substituting $\varepsilon \rightarrow-\varepsilon$ and $\beta \rightarrow-\beta$ we can always suppose

$$
\beta(x) H(t, x)>0, \quad \forall(t, x) \in \Omega .
$$

By (1.7), $\mathcal{R}^{*}, \partial_{u} \mathcal{R}^{*}, \varepsilon \partial_{\varepsilon} \mathcal{R}^{*}$ are continuous in $\bar{\Omega} \times \mathbb{R} \times[-1,1]$ (recall that $H \in E$ ) and

$$
\forall R_{0}>0, \quad\left\|\mathcal{R}^{*}(\cdot ; \varepsilon)\right\|_{C\left(\bar{\Omega} \times\left\{|u| \leqslant R_{0}\right\}\right)},\left\|\partial_{u} \mathcal{R}^{*}(\cdot ; \varepsilon)\right\|_{C\left(\bar{\Omega} \times\left\{|u| \leqslant R_{0}\right\}\right)} \stackrel{\varepsilon \rightarrow 0}{\longrightarrow} 0 .
$$

Moreover, since $H \in H^{1}(\Omega), \partial_{t} \mathcal{R}^{*}=\varepsilon^{-1} \partial_{t} \mathcal{R}+\varepsilon^{1 /(2 k)-1} \partial_{u} \mathcal{R} H_{t}$ and (1.7), then

$$
\left\|\partial_{t} \mathcal{R}^{*}(\cdot, u(\cdot) ; \varepsilon)\right\|_{L^{2}(\Omega)} \leqslant C^{*}\left(\|u\|_{L^{\infty}(\Omega)}\right) \quad \forall u \in L^{\infty}(\Omega),
$$

for a suitable increasing function $C^{*}(\cdot)$.

In order to find solutions of problem (4.1) we perform the Lyapunov-Schmidt reduction of the previous section. We fix $R>0$ to be chosen later (large enough!). Since $f, \partial_{u} f, \varepsilon \partial_{\varepsilon} f$ are continuous on $\bar{\Omega} \times \mathbb{R} \times[-1,1]$, using Proposition 3.2 we solve the range equation (1.14) finding $w=w(v, \varepsilon)$ for $\|v\|_{L^{\infty}}<2 R$ and $|\varepsilon|<\varepsilon_{0}(R)$. Now we look for minimum or maximum points of the corresponding reduced action functional $\Phi$ in $\overline{B_{R}}$ according to whether $\varepsilon>0$ or $\varepsilon<0$. Since $\Phi$ attains minimum or maximum at some point $\bar{v}:=\bar{v}(\varepsilon):=\bar{v}(t, x ; \varepsilon)$ in $\overline{B_{R}}$, to conclude the existence proof of Theorem 2(i) we need to show that $\bar{v}$ is an interior point in $B_{R}$, i.e. $\|\bar{v}\|_{H^{1}}<R$, for a suitable choice of $R$ large enough. Let $\bar{w}:=\bar{w}(t, x ; \varepsilon):=w(\bar{v}(\varepsilon), \varepsilon)(t, x) \in E$ and $\bar{u}:=\bar{u}(t, x ; \varepsilon):=\bar{v}+\bar{w} \in E$. By (3.4) and the definitions of $C_{0}(\cdot), \varepsilon_{0}(\cdot)$ given in Proposition 3.2, we have

$$
\|\bar{w}\|_{E} \leqslant C_{0}(R)|\varepsilon| \leqslant \frac{1}{2}, \quad\|\bar{u}\|_{C(\bar{\Omega})} \leqslant R+\frac{1}{2},
$$

since, by (2.16), $\|\bar{v}\|_{C(\bar{\Omega})} \leqslant\|\bar{v}\|_{H^{1}(\Omega)} \leqslant R$. Let

$$
\mathcal{R}_{*}(t, x ; \varepsilon):=\mathcal{R}^{*}(t, x, \bar{u}(t, x ; \varepsilon) ; \varepsilon) .
$$

We have $\mathcal{R}_{*} \in C(\bar{\Omega})$ and, choosing $R_{0}:=R+1 / 2$ in (4.5),

$$
\left\|\mathcal{R}_{*}(\cdot ; \varepsilon)\right\|_{C(\bar{\Omega})} \stackrel{\varepsilon \rightarrow 0}{\longrightarrow} 0 .
$$

Moreover, since $\partial_{t} \mathcal{R}_{*}=\partial_{t} \mathcal{R}^{*}(\bar{u})+\partial_{u} \mathcal{R}^{*}(\bar{u})\left(\bar{v}_{t}+\bar{w}_{t}\right)$, we have $\partial_{t} \mathcal{R}_{*} \in L^{2}(\Omega)$ with

$$
\left\|\partial_{t} \mathcal{R}_{*}(\cdot ; \varepsilon)\right\|_{L^{2}(\Omega)} \leqslant C_{*}\left(\|\bar{v}\|_{L^{\infty}(\Omega)}\right)+\mathrm{o}(1)\left\|\bar{v}_{t}\right\|_{L^{2}(\Omega)},
$$


for a suitable increasing function $C_{*}(\cdot)$. By (4.2), (4.3) and (4.7), the variational inequality (1.17) yields, for any admissible variation $\varphi \in V$,

$$
0 \geqslant \varepsilon \int_{\Omega} \beta(x)(H+\bar{v}+\bar{w})^{2 k} \varphi+\mathcal{R}_{*} \varphi \quad \forall \varepsilon>0
$$

if $\bar{v}$ is a minimum point, respectively

$$
0 \leqslant \varepsilon \int_{\Omega} \beta(x)(H+\bar{v}+\bar{w})^{2 k} \varphi+\mathcal{R}_{*} \varphi \quad \forall \varepsilon<0
$$

if $\bar{v}$ is a maximum point. However, in both cases we get, dividing by $\varepsilon$,

$$
\int_{\Omega} \beta(x)(H+\bar{v}+\bar{w})^{2 k} \varphi \leqslant \int_{\Omega}-\mathcal{R}_{*} \varphi .
$$

The required a-priori estimate for the $H^{1}$-norm of $\bar{v}$ will be proved in several steps inserting into the variational inequality (4.10) suitable admissible variations. We shall derive, first, an $L^{2 k}$-estimate for $\bar{v}$ (it is needed at least when $k \geqslant 2$ ), see (4.19), next, an $L^{\infty}$-estimate, see (4.33), and, finally, the $H^{1}$-estimate, see (4.45).

The following key estimates will be heavily exploited.

Lemma 4.1. Let $v \in N \cap L^{2 k}(\Omega)$ and $k \in \mathbb{N}^{+}$. Then

$$
\int_{\Omega} v^{2 k} \leqslant \pi 4^{k} \int_{0}^{2 \pi} \hat{v}^{2 k} .
$$

Moreover, for $k=1$

$$
\int_{\Omega_{\alpha}} v^{2} \geqslant 2 \pi[1-4 \alpha] \int_{0}^{2 \pi} \hat{v}^{2} \geqslant \pi \int_{0}^{2 \pi} \hat{v}^{2}
$$

if $0 \leqslant \alpha \leqslant 1 / 8$. For $k \geqslant 2$,

$$
\int_{\Omega_{\alpha}} v^{2 k} \geqslant 2 \pi[1-2(1+2 k) \alpha] \int_{0}^{2 \pi} \hat{v}^{2 k} \geqslant \pi \int_{0}^{2 \pi} \hat{v}^{2 k}
$$

if $0 \leqslant \alpha \leqslant 1 / 4(1+2 k)$.

Proof. From the inequality (2.20) we obtain

$$
\int_{\Omega} v^{2 k}=\int_{\Omega}\left(v_{+}-v_{-}\right)^{2 k} \leqslant 2^{2 k-1} \int_{\Omega} v_{+}^{2 k}+v_{-}^{2 k}=2^{2 k-1} \int_{\Omega} \hat{v}^{2 k}(t+x)+\hat{v}^{2 k}(t-x) \mathrm{d} t \mathrm{~d} x,
$$

which, using (2.11), proves (4.11).

We now prove the second part of the lemma in the case $k \geqslant 2$. Using the inequality (2.21), (2.11) and (2.12), we obtain

$$
\begin{aligned}
\int_{\Omega_{\alpha}} v^{2 k} & =\int_{\Omega_{\alpha}}\left(v_{+}-v_{-}\right)^{2 k} \geqslant \int_{\Omega_{\alpha}} v_{+}^{2 k}+v_{-}^{2 k}-2 k \int_{\Omega_{\alpha}} v_{+}^{2 k-1} v_{-}+v_{+} v_{-}^{2 k-1} \\
& =2 \pi(1-2 \alpha) \int_{0}^{2 \pi} \hat{v}^{2 k}-4 k \int_{\Omega_{\alpha}} v_{+}^{2 k-1} v_{-} .
\end{aligned}
$$

By (2.10) and since $\hat{v}$ has zero average 


$$
\left|\int_{\Omega_{\alpha}} v_{+}^{2 k-1} v_{-}\right|=\frac{1}{2}\left|\int_{-2 \pi \alpha}^{2 \pi \alpha} \int_{0}^{2 \pi} \hat{v}^{2 k-1}(y) \hat{v}(y+z) \mathrm{d} y \mathrm{~d} z\right| \leqslant \frac{1}{2} \int_{-2 \pi \alpha}^{2 \pi \alpha} \int_{0}^{2 \pi}|\hat{v}(y)|^{2 k-1}|\hat{v}(y+z)| \mathrm{d} y \mathrm{~d} z .
$$

By Hölder inequality with $p:=2 k /(2 k-1)$ and $q:=2 k(1 / p+1 / q=1)$,

$$
\int_{0}^{2 \pi}|\hat{v}(y)|^{2 k-1}|\hat{v}(y+z)| \mathrm{d} y \leqslant\left(\int_{0}^{2 \pi}|\hat{v}(y)|^{2 k} \mathrm{~d} y\right)^{(2 k-1) /(2 k)}\left(\int_{0}^{2 \pi}|\hat{v}(y+z)|^{2 k} \mathrm{~d} y\right)^{1 /(2 k)}=\int_{0}^{2 \pi}|\hat{v}(y)|^{2 k} \mathrm{~d} y,
$$

where, in the equality, we have used the periodicity of $\hat{v}$ to conclude that $\int_{0}^{2 \pi}|\hat{v}(y+z)|^{2 k} \mathrm{~d} y=\int_{0}^{2 \pi}|\hat{v}(y)|^{2 k} \mathrm{~d} y$. Inserting the last inequality in (4.13), we obtain

$$
\left|\int_{\Omega_{\alpha}} v_{+}^{2 k-1} v_{-}\right| \leqslant 2 \pi \alpha \int_{0}^{2 \pi}|\hat{v}(y)|^{2 k} \mathrm{~d} y .
$$

Inserting (4.14) in (4.12) the proposition follows in the case $k \geqslant 2$. The case $k=1$ is similar:

$$
\int_{\Omega_{\alpha}} v^{2}=\int_{\Omega_{\alpha}} v_{+}^{2}+v_{-}^{2}-2 v_{+} v_{-}=2 \pi(1-2 \alpha) \int_{0}^{2 \pi} \hat{v}^{2}-2 \int_{\Omega_{\alpha}} v_{+} v_{-}
$$

and we conclude by (4.14).

We get as corollary the following key proposition.

Proposition 4.2. Let $k \in \mathbb{N}^{+}$and $B \in C(\bar{\Omega})$ with $B \geqslant 0$ in $\Omega$. Define

$$
c_{k}(B):=\frac{1}{4^{k}} \min _{\bar{\Omega}_{\alpha_{k}}} B \quad \text { where } \alpha_{1}:=\frac{1}{8}, \alpha_{k}:=\frac{1}{4(1+2 k)} \text { for } k \geqslant 2 .
$$

Then $\forall v \in N \cap L^{2 k}(\Omega)$

$$
\int_{\Omega} B v^{2 k} \geqslant c_{k}(B) \int_{\Omega} v^{2 k}
$$

Proof. Since $B \geqslant 0$ in $\Omega$ and using Lemma 4.1

$$
\int_{\Omega} B v^{2 k} \geqslant \min _{\bar{\Omega}_{\alpha_{k}}} B \int_{\Omega_{\alpha_{k}}} v^{2 k} \geqslant \pi \min _{\bar{\Omega}_{\alpha_{k}}} B \int_{0}^{2 \pi} \hat{v}^{2 k} \geqslant \frac{1}{4^{k}} \min _{\bar{\Omega}_{\alpha_{k}}} B \int_{\Omega} v^{2 k} .
$$

Remark 4.3. We stress that estimate (4.16) is not trivial (in the case $c_{k}(B)>0$ ) since $B$ could vanish on $\partial \Omega$ (in particular, in this case, (4.16) does not hold true in the whole $\left.L^{2 k}(\Omega)\right)$.

Remark 4.4. In light of Proposition 4.2 we can prove Theorem 2 requiring only $H \geqslant 0$ in $\Omega$ and $H>0$ in $\bar{\Omega}_{\alpha_{k}}$, instead of (1.8).

In the following $\kappa_{i}$ will denote positive constants depending only on $H, \beta, \mathcal{R}, k$ but not on $R, \varepsilon$. We also recall the notation o(1) for a function tending to 0 as $\varepsilon \rightarrow 0$ (possibly depending on $R$ ).

\subsection{The $L^{2 k}$-estimate}

Take $\varphi:=\bar{v}$ in the variational inequality (4.10); $\varphi$ is an admissible variation since $\langle\bar{v}, \varphi\rangle_{H^{1}}=\|\bar{v}\|_{H^{1}}^{2}>0$. 
By (4.10), (4.8) and $\|w(\bar{v}, \varepsilon)\|_{E} \leqslant C_{0}(R)|\varepsilon|$ (recall (4.6)), we get

$$
\int_{\Omega} \beta(\bar{v}+H)^{2 k} \bar{v} \leqslant \int_{\Omega}\left|\mathcal{R}_{*} \bar{v}\right|+\left|\beta(H+\bar{v}+\bar{w})^{2 k}-\beta(H+\bar{v})^{2 k}\right||\bar{v}| \leqslant \mathrm{o}(1)\|\bar{v}\|_{L^{1}} \leqslant \mathrm{o}(1) R
$$

and therefore there exists $0<\varepsilon_{1}(R) \leqslant \varepsilon_{0}(R)$ such that

$$
\int_{\Omega} \beta(\bar{v}+H)^{2 k} \bar{v} \leqslant 1 \quad \text { for }|\varepsilon| \leqslant \varepsilon_{1}(R) .
$$

Noting that, $\int_{\Omega} \beta \bar{v}^{2 k+1}=0$ by (2.18) with $a(x, u)=\beta(x) u^{2 k}, \beta(\pi-x)=\beta(x)$, and using Proposition 4.2, we derive

$$
\begin{aligned}
\int_{\Omega} \beta(\bar{v}+H)^{2 k} \bar{v} & =\int_{\Omega} \beta\left[(\bar{v}+H)^{2 k}-\bar{v}^{2 k}\right] \bar{v}=\int_{\Omega} 2 k \beta H \bar{v}^{2 k}+\beta \sum_{j=0}^{2 k-2}\left(\begin{array}{c}
2 k \\
j
\end{array}\right) \bar{v}^{j+1} H^{2 k-j} \\
& \geqslant 2 k c_{k}(\beta H)\|\bar{v}\|_{L^{2 k}}^{2 k}-\kappa_{1}\|\bar{v}\|_{L^{2 k}}^{2 k-1}-\kappa_{2}\|\bar{v}\|_{L^{2 k}},
\end{aligned}
$$

where $c_{k}(\beta H)>0$ was defined in (4.15) (recall that $\beta H>0$ by (4.4)) and we have used Hölder inequality to estimate $\|\bar{v}\|_{L^{i}} \leqslant C_{i, k}\|\bar{v}\|_{L^{2 k}}(i \leqslant 2 k-1)$.

Finally, by (4.17) and (4.18) we deduce

$$
\|\bar{v}\|_{L^{2 k}} \leqslant \kappa_{3} \text { for }|\varepsilon| \leqslant \varepsilon_{1}(R) \text {. }
$$

\subsection{The $L^{\infty}$-estimate}

To obtain the $L^{\infty}$-estimate for $\bar{v}$ we consider an admissible variation $\varphi$ constructed as in [18]. Let, for $M>0$,

$$
q(\lambda):=q_{M}(\lambda):= \begin{cases}0, & \text { if }|\lambda| \leqslant M \\ \lambda-M, & \text { if } \lambda \geqslant M \\ \lambda+M, & \text { if } \lambda \leqslant M\end{cases}
$$

For $\bar{v}(t, x)=\bar{v}_{+}(t, x)-\bar{v}_{-}(t, x)=\hat{\bar{v}}(t+x)-\hat{\bar{v}}(t-x)$, we define

$$
\varphi:=q_{+}-q_{-}:=q\left(\bar{v}_{+}\right)-q\left(\bar{v}_{-}\right) \in V .
$$

We take

$$
M:=\frac{1}{2}\|\hat{\bar{v}}\|_{L^{\infty}(\mathbb{T})}
$$

and we can assume $M>0$, i.e. $\bar{v}$ is not identically zero.

In [18] it is proved that such $\varphi$ is an admissible variation. We report the proof for completeness. By (3.16), it is sufficient to prove that $\langle\bar{v}, \varphi\rangle_{H^{1}}>0$.

Using (2.10), (2.11) and (2.12)

$$
\left\langle\bar{v}_{+}-\bar{v}_{-}, q\left(\bar{v}_{+}\right)-q\left(\bar{v}_{-}\right)\right\rangle_{H^{1}}=\int_{\Omega} \bar{v}_{+} q\left(\bar{v}_{+}\right)+\bar{v}_{-} q\left(\bar{v}_{-}\right)+2 \int_{\Omega} q^{\prime}\left(\bar{v}_{+}\right)\left[\left(\bar{v}_{+}\right)_{t}^{2}+\left(\bar{v}_{+}\right)_{x}^{2}\right] .
$$

Since $q$ is a monotone odd function of its argument and by our choice of $M, \bar{v}_{ \pm} q\left(\bar{v}_{ \pm}\right)>0$ in a positive measure set, and, since $q^{\prime} \geqslant 0$, the second term in (4.21) is non-negative.

We also have, since $q$ is a monotone function,

$$
\bar{v} \varphi=\left(\bar{v}_{+}-\bar{v}_{-}\right)\left(q_{+}-q_{-}\right)=\left(\bar{v}_{+}-\bar{v}_{-}\right)\left(q\left(\bar{v}_{+}\right)-q\left(\bar{v}_{-}\right)\right) \geqslant 0 .
$$

Insert such $\varphi$ in the variational inequality (4.10). Here the dominant term is $\int \beta(\bar{v}+H)^{2 k} \varphi$, in the sense that, by $\|w(v, \varepsilon)\|_{E} \leqslant C_{0}(R)|\varepsilon|$ (recall (4.6)), (4.8) and $\|\bar{v}\|_{L^{\infty}} \leqslant R$, we obtain that there exists $0<\varepsilon_{2}(R) \leqslant \varepsilon_{1}(R)$ such that

$$
\int_{\Omega} \beta(\bar{v}+H)^{2 k} \varphi \leqslant\|\varphi\|_{L^{1}} \quad \text { for }|\varepsilon| \leqslant \varepsilon_{2}(R) .
$$


Since $\int_{\Omega} \beta \bar{v}^{2 k} \varphi=0$ by (2.18) and $\beta(\pi-x)=\beta(x)$, we have (recall $\beta>0$ )

$$
\int_{\Omega} \beta(\bar{v}+H)^{2 k} \varphi=\int_{\Omega} \beta\left[(\bar{v}+H)^{2 k}-\bar{v}^{2 k}\right] \varphi \geqslant \int_{\Omega} 2 k \beta H \bar{v}^{2 k-1} \varphi-\kappa_{4}\left(\|\bar{v}\|_{L^{\infty}}^{2 k-2}+1\right)\|\varphi\|_{L^{1}} .
$$

We now estimate the dominant term $2 k \int_{\Omega} \beta H \bar{v}^{2 k-1} \varphi$. Since $\bar{v} \varphi \geqslant 0$ and $\min _{\Omega_{1 / 4}} \beta H>0$ (by (4.4))

$$
\int_{\Omega} 2 k \bar{v}^{2 k-1} \beta H \varphi=2 k \int_{\Omega} \beta H(\bar{v} \varphi) \bar{v}^{2 k-2} \geqslant \kappa_{5} \int_{\Omega_{1 / 4}}(\bar{v} \varphi) \bar{v}^{2 k-2} .
$$

By (4.23), (4.24) and (4.25), we have

$$
\int_{\Omega_{1 / 4}} \bar{v}^{2 k-1} \varphi \leqslant \kappa_{6}\left(\|\bar{v}\|_{L^{\infty}(\Omega)}^{2 k-2}+1\right)\|\varphi\|_{L^{1}(\Omega)} .
$$

We have to give a lower bound of the positive integral $\int_{\Omega_{1 / 4}} \bar{v}^{2 k-1} \varphi=\int_{\Omega_{1 / 4}}(\bar{v} \varphi) \bar{v}^{2 k-2}=\int_{\Omega_{1 / 4}}(\bar{v} \varphi)\left(\bar{v}_{+}-\bar{v}_{-}\right)^{2 k-2}$.

We first consider the (more difficult) case $k \geqslant 2$, in which the $L^{2 k}$-estimate for $\bar{v}$ obtained in the previous subsection is needed, the (simpler) case $k=1$ will be treated later.

Using (2.21) we obtain

$$
\begin{aligned}
\int_{\Omega_{1 / 4}} \bar{v}^{2 k-1} \varphi \geqslant & \int_{\Omega_{1 / 4}} \bar{v} \varphi\left[\bar{v}_{+}^{2 k-2}+\bar{v}_{-}^{2 k-2}-(2 k-2)\left(\bar{v}_{+}^{2 k-3} \bar{v}_{-}+\bar{v}_{+} \bar{v}_{-}^{2 k-3}\right)\right] \\
= & 2 \int_{\Omega_{1 / 4}} \bar{v}_{+}^{2 k-1} q_{+}-\bar{v}_{+}^{2 k-1} q_{-}+\bar{v}_{+}^{2 k-2} \bar{v}_{-} q_{-}-\bar{v}_{+}^{2 k-2} \bar{v}_{-} q_{+} \\
& +(2 k-2)\left[-\bar{v}_{+}^{2 k-2} \bar{v}_{-} q_{+}+\bar{v}_{+}^{2 k-2} \bar{v}_{-} q_{-}-\bar{v}_{+}^{2 k-3} \bar{v}_{-}^{2} q_{-}+\bar{v}_{+}^{2 k-3} \bar{v}_{-}^{2} q_{+}\right] \\
\geqslant & 2 \int_{\Omega_{1 / 4}} \bar{v}_{+}^{2 k-1} q_{+} \\
& -2 \int_{\Omega_{1 / 4}} \bar{v}_{+}^{2 k-1} q_{-}+(2 k-1) \bar{v}_{+}^{2 k-2} \bar{v}_{-} q_{+}+(2 k-2) \bar{v}_{+}^{2 k-3} \bar{v}_{-}^{2} q_{-}
\end{aligned}
$$

where in the equality we have used (2.12) and in the last inequality the fact that $\bar{v}_{+} q_{+}, \bar{v}_{-} q_{-} \geqslant 0$ (since $\lambda q(\lambda) \geqslant 0$ ) and so $\bar{v}_{+}^{2 k-2} \bar{v}_{-} q_{-}, \bar{v}_{+}^{2 k-3} \bar{v}_{-}^{2} q_{+} \geqslant 0$.

The dominant term is (4.27). Since $\lambda^{2 k-1} q(\lambda) \geqslant M^{2 k-1}|q(\lambda)|$, by (2.11) we obtain

$$
2 \int_{\Omega_{1 / 4}} \bar{v}_{+}^{2 k-1} q_{+}=2 \pi\left(1-\frac{2}{4}\right) \int_{0}^{2 \pi} \hat{\bar{v}}^{2 k-1}(s) q(\hat{\bar{v}}(s)) \mathrm{d} s \geqslant \pi M^{2 k-1}\|q(\hat{\bar{v}})\|_{L^{1}(\mathbb{T})} .
$$

We now give an upper estimate of the three terms in (4.27). By (2.10)

$$
\begin{aligned}
& \left|2 \int_{\Omega_{1 / 4}} \bar{v}_{+}^{2 k-1} q_{-}\right| \leqslant 2 \int_{\Omega}\left|\bar{v}_{+}^{2 k-1}\right|\left|q_{-}\right| \leqslant\|\hat{\bar{v}}\|_{L^{2 k-1}(\mathbb{T})}^{2 k-1}\|q(\hat{\bar{v}})\|_{L^{1}(\mathbb{T})}, \\
& \left|2 \int_{\Omega_{1 / 4}}\left(\bar{v}_{+}^{2 k-2} q_{+}\right) \bar{v}_{-}\right| \leqslant\left\|\hat{\bar{v}}^{2 k-2} q(\hat{\bar{v}})\right\|_{L^{1}(\mathbb{T})}\|\hat{\bar{v}}\|_{L^{1}(\mathbb{T})} \leqslant(2 M)^{2 k-2}\|q(\hat{\bar{v}})\|_{L^{1}(\mathbb{T})}\|\hat{\bar{v}}\|_{L^{1}(\mathbb{T})}, \\
& \left|2 \int_{\Omega_{1 / 4}} \bar{v}_{+}^{2 k-3}\left(\bar{v}_{-}^{2} q_{-}\right)\right| \leqslant\left\|\hat{\bar{v}}^{2 k-3}\right\|_{L^{1}(\mathbb{T})}\left\|\hat{\bar{v}}^{2} q(\hat{\bar{v}})\right\|_{L^{1}(\mathbb{T})} \leqslant(2 M)^{2}\|\hat{\bar{v}}\|_{L^{2 k-3}(\mathbb{T})}^{2 k-3}\|q(\hat{\bar{v}})\|_{L^{1}(\mathbb{T})} .
\end{aligned}
$$


By the previous inequalities, (4.29), Hölder inequality ${ }^{5}$ and using the $L^{2 k}$-estimate (4.19) for $\bar{v}$ obtained in the previous subsection, we finally have

$$
\int_{\Omega_{1 / 4}} \bar{v}^{2 k-1} \varphi \geqslant \pi M^{2 k-1}\|q(\hat{\bar{v}})\|_{L^{1}(\mathbb{T})}-\kappa_{7}\left(M^{2 k-2}+1\right)\|q(\hat{\bar{v}})\|_{L^{1}(\mathbb{T})} .
$$

Now we note that by $(2.11)$

$$
\int_{\Omega}|\varphi| \leqslant \int_{\Omega}\left|q\left(v_{+}\right)\right|+\left|q\left(v_{-}\right)\right|=2 \pi\|q(\hat{\bar{v}})\|_{L^{1}(\mathbb{T})} .
$$

We collect (4.26) and (4.30) using (4.31) in order to obtain

$$
M^{2 k-1}\|q(\hat{\bar{v}})\|_{L^{1}(\mathbb{T})} \leqslant \kappa_{8}\left(\|\bar{v}\|_{L^{\infty}(\Omega)}^{2 k-2}+M^{2 k-2}+1\right)\|q(\hat{\bar{v}})\|_{L^{1}(\mathbb{T})} .
$$

Since $M:=\|\hat{\bar{v}}\|_{L^{\infty}(\mathbb{T})} / 2$ hence $\|q(\hat{\bar{v}})\|_{L^{\infty}(\mathbb{T})}=M,\|\bar{v}\|_{L^{\infty}(\Omega)} \leqslant 2\|\hat{\bar{v}}\|_{L^{\infty}(\mathbb{T})}=4 M$ and $\|\hat{\bar{v}}\|_{L^{\infty}(\mathbb{T})} \neq 0$. Hence, by (4.32),

$$
M^{2 k-1}\|q(\hat{\bar{v}})\|_{L^{1}(\mathbb{T})} \leqslant \kappa_{9}\left(M^{2 k-2}+1\right)\|q(\hat{\bar{v}})\|_{L^{1}(\mathbb{T})}
$$

and, dividing by $\|q(\hat{\bar{v}})\|_{L^{1}(\mathbb{T})} \neq 0$, we finally obtain $M^{2 k-1} \leqslant \kappa_{9}\left(M^{2 k-2}+1\right)$.

By our choice of $M$ the $L^{\infty}$-estimate follows for $k \geqslant 2$,

$$
\|\bar{v}\|_{L^{\infty}} \leqslant \kappa_{10} \text { for }|\varepsilon| \leqslant \varepsilon_{2}(R) .
$$

We now briefly discuss the case $k=1$, which is simpler and where a previous $L^{2}$-estimate for $\bar{v}$ is not necessary to obtain (4.33). In fact by (4.23) and (4.24) (with $k=1$ ), we obtain

$$
\int_{\Omega} \beta H \bar{v} \varphi \leqslant \kappa_{11}\|\varphi\|_{L^{1}}
$$

For $0<\alpha<1 / 2$ to be chosen later, we have

$$
\int_{\Omega} \beta H \bar{v} \varphi \geqslant \min _{\bar{\Omega}_{\alpha}}(\beta H) \int_{\Omega_{\alpha}} \bar{v} \varphi
$$

We have to give a lower bound of

$$
\int_{\Omega_{\alpha}} \bar{v} \varphi=\int_{\Omega_{\alpha}} \bar{v}_{+} q_{+}+\bar{v}_{-} q_{-}-\int_{\Omega_{\alpha}} \bar{v}_{+} q_{-}+\bar{v}_{-} q_{+} .
$$

By (2.11) and $\lambda q(\lambda) \geqslant M|q(\lambda)|$

$$
\int_{\Omega_{\alpha}} \bar{v}_{+} q_{+}=\int_{\Omega_{\alpha}} \bar{v}_{-} q_{-}=\pi(1-2 \alpha) \int_{0}^{2 \pi} \hat{\bar{v}}(s) q(\hat{\bar{v}}(s)) \mathrm{d} s \geqslant \pi(1-2 \alpha) M\|q(\hat{\bar{v}})\|_{L^{1}(\mathbb{T})} .
$$

Moreover, since $\hat{\bar{v}}$ has zero average, by (2.10), we have

$$
\left|\int_{\Omega_{\alpha}} \bar{v}_{+} q_{-}\right|=\left|\int_{\Omega_{\alpha}} \bar{v}_{-} q_{+}\right| \leqslant \frac{1}{2} \int_{-2 \alpha \pi}^{2 \alpha \pi} \int_{0}^{2 \pi}|q(\hat{\bar{v}}(y)) \hat{\bar{v}}(z+y)| \mathrm{d} y \mathrm{~d} z \leqslant 2 \alpha \pi M\|q(\hat{\bar{v}})\|_{L^{1}(\mathbb{T})} .
$$

Collecting (4.36), (4.37) and (4.38) we obtain

$$
\int_{\Omega_{\alpha}} \bar{v} \varphi \geqslant 2 \pi(1-6 \alpha) M\|q(\hat{\bar{v}})\|_{L^{1}(\mathbb{T})} \geqslant \frac{1}{2} \pi M\|q(\hat{\bar{v}})\|_{L^{1}(\mathbb{T})},
$$

\footnotetext{
$\overline{5}$ To estimate $\|\hat{\bar{v}}\|_{L^{j}(\mathbb{T})} \leqslant C_{j, k}\|\hat{\hat{v}}\|_{L^{2 k}(\mathbb{T})}$ for $j<2 k$.
} 
choosing $\alpha:=1 / 8$. Collecting (4.34), (4.35) and (4.39), we obtain

$$
M\|q(\hat{\bar{v}})\|_{L^{1}(\mathbb{T})} \leqslant \kappa_{12}\|\varphi\|_{L^{1}(\Omega)} .
$$

Using (4.31) and dividing by $\|q(\hat{\bar{v}})\|_{L^{1}(\mathbb{T})} \neq 0$ in the previous inequality we finally obtain (4.33) also in the case $k=1$.

\subsection{The $H^{1}$-estimate}

We note that $\varphi:=-D_{-h} D_{h} \bar{v}$ is an admissible variation, since using (2.26),

$$
\left\langle-D_{-h} D_{h} \bar{v}, \bar{v}\right\rangle_{H^{1}}=\left\langle D_{h} \bar{v}, D_{h} \bar{v}\right\rangle_{H^{1}}>0 .
$$

Since $\partial_{t}\left[\beta(H+\bar{v}+\bar{w})^{2 k}\right], \partial_{t} \mathcal{R}_{*} \in L^{2}(\Omega)$ (see (4.9)), we have, as $h \rightarrow 0$,

$$
\begin{aligned}
& \int_{\Omega} \beta(H+\bar{v}+\bar{w})^{2 k} \varphi \stackrel{(2.26)}{=} \int_{\Omega} \beta D_{h}\left[(H+\bar{v}+\bar{w})^{2 k}\right] D_{h} \bar{v} \stackrel{(2.29)}{\longrightarrow} \int_{\Omega} \beta\left[(H+\bar{v}+\bar{w})^{2 k}\right]_{t} \bar{v}_{t}, \\
& \int_{\Omega} \mathcal{R}_{*} \varphi \stackrel{(2.26)}{=} \int_{\Omega} D_{h} \mathcal{R}_{*} D_{h} \bar{v} \stackrel{(2.29)}{\longrightarrow} \int_{\Omega} \partial_{t} \mathcal{R}_{*} \bar{v}_{t},
\end{aligned}
$$

and, by the variational inequality (4.10), we obtain

$$
\int_{\Omega} \beta\left[(H+\bar{v}+\bar{w})^{2 k}\right]_{t} \bar{v}_{t} \leqslant \int_{\Omega}-\partial_{t} \mathcal{R}_{*} \bar{v}_{t} .
$$

By the $L^{\infty}$-estimate on $\bar{v}$ given in (4.33), the Cauchy-Schwartz inequality and (4.9) we obtain

$$
\left|\int_{\Omega} \partial_{t} \mathcal{R}_{*} \bar{v}_{t}\right| \leqslant \kappa_{13}\left\|\bar{v}_{t}\right\|_{L^{2}}+\mathrm{o}(1)\left\|\bar{v}_{t}\right\|_{L^{2}}^{2}
$$

Since $\|\bar{w}\|_{E}=\|w(\bar{v}, \varepsilon)\|_{E} \leqslant C_{0}(R)|\varepsilon|$ (recall (4.6)), again by (4.33) and the Cauchy-Schwartz inequality, we find

$$
\begin{aligned}
\int_{\Omega} \beta\left[(H+\bar{v}+\bar{w})^{2 k}\right]_{t} \bar{v}_{t} & =2 k \int_{\Omega} \beta(H+\bar{v}+\bar{w})^{2 k-1}\left(H_{t}+\bar{v}_{t}+\bar{w}_{t}\right) \bar{v}_{t} \\
& \geqslant 2 k \int_{\Omega} \beta(\bar{v}+H)^{2 k-1} \bar{v}_{t}^{2}-\mathrm{o}(1)\left\|\bar{v}_{t}\right\|_{L^{2}}^{2}-\kappa_{14}\left\|\bar{v}_{t}\right\|_{L^{2}} .
\end{aligned}
$$

Collecting (4.40), (4.41) and (4.42), we obtain

$$
\int_{\Omega} \beta(\bar{v}+H)^{2 k-1} \bar{v}_{t}^{2} \leqslant \kappa_{15}\left\|\bar{v}_{t}\right\|_{L^{2}}+\mathrm{o}(1)\left\|\bar{v}_{t}\right\|_{L^{2}}^{2} .
$$

Since $\bar{v}, \bar{v}_{t} \in N$ and $\bar{v}^{2 k-1} \bar{v}_{t}^{2} \in L^{1}(\Omega)$, it results $\int_{\Omega} \beta \bar{v}^{2 k-1} \bar{v}_{t}^{2}=0$ by (2.19). Using the inequality (2.22) we obtain

$$
\int_{\Omega} \beta(\bar{v}+H)^{2 k-1} \bar{v}_{t}^{2}=\int_{\Omega} \beta\left[(\bar{v}+H)^{2 k-1}-\bar{v}^{2 k-1}\right] \bar{v}_{t}^{2} \geqslant 4{ }^{1-k} \int_{\Omega} \beta H^{2 k-1} \bar{v}_{t}^{2} \geqslant 4^{1-k} c_{1}\left(\beta H^{2 k-1}\right) \int_{\Omega} \bar{v}_{t}^{2}
$$

where $c_{1}(\cdot)$ was defined in (4.15) and $\beta H^{2 k-1}>0$ by (4.4). By (4.43) and (4.44) we get

$$
\left\|\bar{v}_{t}\right\|_{L^{2}}^{2} \leqslant \kappa_{16}\left\|\bar{v}_{t}\right\|_{L^{2}}+\mathrm{o}(1)\left\|\bar{v}_{t}\right\|_{L^{2}}^{2}
$$

and we finally deduce that there exists a $0<\varepsilon_{3}(R) \leqslant \varepsilon_{2}(R)$ such that

$$
\|\bar{v}\|_{H^{1}}<\kappa_{17} \quad \forall|\varepsilon| \leqslant \varepsilon_{3}(R) .
$$

Proof of Theorem 2(i) completed. Defining $R_{*}:=\kappa_{17}$ and $\varepsilon_{*}:=\varepsilon_{3}\left(R_{*}\right)$ we obtain, by (4.45), that

$$
\|\bar{v}(\varepsilon)\|_{H^{1}}<R_{*} \quad \forall|\varepsilon| \leqslant \varepsilon_{*}
$$


and $\bar{v}(\varepsilon)$ is an interior minimum or maximum point of $\Phi$ in $B_{R_{*}}:=\left\{\|v\|_{H^{1}}<R_{*}\right\}$. By Lemma $3.3 \bar{u}=\bar{v}+\bar{w}=$ $\bar{v}(\varepsilon)+w(\bar{v}(\varepsilon), \varepsilon)$ is a weak solution of $(4.1)$ and

$$
u:=\varepsilon\left(H+\bar{v}\left(\varepsilon^{2 k}\right)+w\left(\bar{v}\left(\varepsilon^{2 k}\right), \varepsilon^{2 k}\right)\right) \in E
$$

is a weak solution of (1.1)-(1.3) satisfying $\|u\|_{E} \leqslant C|\varepsilon|$.

Remark 4.5. Let $u:=u_{\varepsilon}=v_{\varepsilon}+w_{\varepsilon}, v_{\varepsilon} \in V, w_{\varepsilon} \in W$, be a weak solution of $\square u_{\varepsilon}=\varepsilon\left(g\left(x, u_{\varepsilon}\right)+h(t, x)\right)$ where $g \in C([0, \pi] \times \mathbb{R}), g(x, u)=g(x,-u)=g(\pi-x, u)$. Suppose $u_{\varepsilon}$ satisfies $\left\|u_{\varepsilon}\right\|_{L^{\infty}} \leqslant R, \forall \varepsilon$ small. We claim this implies $h \in N^{\perp}$. Indeed, $w_{\varepsilon}$ satisfies the range equation $w_{\varepsilon}=\varepsilon \square^{-1} \Pi_{N^{\perp}}\left(g\left(x, u_{\varepsilon}\right)+h(t, x)\right)$ and therefore $\left\|w_{\varepsilon}\right\|_{L^{\infty}} \leqslant$ $C(R)|\varepsilon|$. Moreover, by the kernel equation $\Pi_{N}\left(g\left(x, v_{\varepsilon}+w_{\varepsilon}\right)+h(t, x)\right)=0$, and noting that $\Pi_{N} g\left(x, v_{\varepsilon}\right)=0$ by (2.18), we derive

$$
\left\|\Pi_{N} h(t, x)\right\|_{L^{2}}=\left\|\Pi_{N}\left(g\left(x, v_{\varepsilon}+w_{\varepsilon}\right)-g\left(x, v_{\varepsilon}\right)\right)\right\|_{L^{2}} \leqslant\left\|g\left(x, v_{\varepsilon}+w_{\varepsilon}\right)-g\left(x, v_{\varepsilon}\right)\right\|_{L^{2}} \rightarrow 0
$$

as $\left\|w_{\varepsilon}\right\|_{L^{\infty}} \rightarrow 0$ because $g$ is uniformly continuous on any compact set $[0, \pi] \times\{|u| \leqslant C\}$. Therefore $\Pi_{N} h=0$ and $h \in N^{\perp}$.

Remark 4.6 ((Multiplicity)). By (2.18), any forcing term $h(t, x):=-g\left(x, v_{0}(t, x)\right), v_{0} \in V \backslash\{0\}$, is in $N^{\perp}$, if $g(x, u)=g(x,-u)=g(\pi-x, u)$. Therefore the equation $\square u=\varepsilon(g(x, u)+h(t, x))$ possesses, beyond the $\varepsilon$-small solution $u$ of Theorem 2, also the other two (not small) solutions $\pm v_{0}$.

Remark 4.7 ((Minimal period)). By (4.46) it is sufficient to prove that $\Pi_{N^{\perp}} u=\varepsilon\left(\square^{-1} h+w\right)$ has minimal period $2 \pi$ w.r.t. time. Note, first, that if $h$ has minimal period $2 \pi$ then so has $\square^{-1} h$. Therefore, since $w$ is small with $\varepsilon$, also $\square^{-1} h+w$ has minimal period $2 \pi$ for $\varepsilon$ small. This follows by the characterization (see Appendix A for a proof)

$$
f \in L^{2}(\Omega) \text { has minimal period } 2 \pi \quad \Longleftrightarrow \quad \exists p_{0}, p_{1}, \ldots, p_{k} \in D \text { with } \operatorname{gcd}\left(p_{0}, p_{1}, \ldots, p_{k}\right)=1
$$

where $D:=\left\{l \in \mathbb{N}^{+} \mid f_{l}(x) \not \equiv 0\right\}$ and $f(t, x)=\sum_{l \in \mathbb{Z}} f_{l}(x) \mathrm{e}^{\mathrm{i} l t}$.

\subsection{Higher regularity and classical solutions}

We now prove Theorem 2(ii) obtaining more regularity for the weak solution $u \in E$ of (1.1)-(1.3) defined in (4.46). Since $\bar{v} \in V:=N \cap H^{1}$ is a critical point of $\Phi: V \rightarrow \mathbb{R}$

$$
\int_{\Omega}\left(\beta(H+\bar{u})^{2 k}+\mathcal{R}_{*}\right) \psi=0 \quad \forall \psi \in N \cap H^{1},
$$

which actually holds for any $\psi \in N$ since $N \cap H^{1}$ is dense in $N$ with the $L^{2}$-topology ${ }^{6}$. Hence, taking $\psi:=\varphi_{t}$ for any $\varphi \in N \cap H^{1}$ in (4.48) and integrating by parts, we find

$$
0=\int_{\Omega} \partial_{t}\left(\beta(H+\bar{v}+\bar{w})^{2 k}+\mathcal{R}_{*}\right) \varphi=\int_{\Omega}\left[2 k \beta(H+\bar{u})^{2 k-1}\left(H_{t}+\bar{v}_{t}+\bar{w}_{t}\right)+\partial_{t} \mathcal{R}_{*}\right] \varphi
$$

for any $\varphi \in N \cap H^{1}$. Since the term into square brackets [.] in (4.49) is in $L^{2}(\Omega)$ then, again by the $L^{2}$-density of $N \cap H^{1}$ in $N$, (4.49) actually holds for any $\varphi \in N$.

Setting for brevity

$$
z:=z(t, x ; \varepsilon):=\left(t, x, \varepsilon^{1 /(2 k)}(H(t, x)+\bar{u}(t, x ; \varepsilon))\right),
$$

we can write, from (4.7), (4.3),

$$
\mathcal{R}_{*}(t, x ; \varepsilon)=\varepsilon^{-1} \mathcal{R}(z) \quad \text { and } \quad \partial_{t} \mathcal{R}_{*}=\varepsilon^{-1+1 /(2 k)} \partial_{u} \mathcal{R}(z) \bar{v}_{t}+A
$$

where

$$
A(t, x ; \varepsilon):=\varepsilon^{-1} \partial_{t} \mathcal{R}(z)+\varepsilon^{-1+1 /(2 k)} \partial_{u} \mathcal{R}(z)\left(H_{t}(t, x)+\bar{w}_{t}(t, x ; \varepsilon)\right) .
$$

${ }^{6}$ Recall that $\left[\beta(H+\bar{u})^{2 k}+\mathcal{R}_{*}\right] \in L^{2}(\Omega)$ since $\beta, H, \bar{u}, \mathcal{R}_{*}$ are continuous functions. 
Then (4.49) becomes

$$
\int_{\Omega}\left[2 k \beta(H+\bar{u})^{2 k-1}\left(H_{t}+\bar{v}_{t}+\bar{w}_{t}\right)+\varepsilon^{-1+1 /(2 k)} \partial_{u} \mathcal{R}(z) \bar{v}_{t}+A\right] \varphi=0 \quad \forall \varphi \in N .
$$

For the remainder of this subsection we shall take $\varepsilon \neq 0$, and $K_{i}$ will denote suitable positive constants possibly depending ${ }^{7}$ also on $\varepsilon$.

Since we are assuming that $h \in H^{j} \cap C^{j-1}, j \geqslant 1$, then, by (2.3), $H \in H^{j+1} \cap C^{j}$. Hence, to prove that $u \in$ $H^{j+1} \cap C^{j}$, by (4.46), it is sufficient to show that $\bar{v}, \bar{w} \in H^{j+1} \cap C^{j}$.

We first prove that

Lemma 4.8. $\bar{u} \in C^{1}(\bar{\Omega}) \cap H^{2}(\Omega)$.

Proof. We shall divide the proof in three steps.

Step 1: $\bar{w} \in C^{1}(\bar{\Omega}) \cap H^{2}(\Omega)$ and

$$
\|\bar{w}\|_{C^{1}}+\|\bar{w}\|_{H^{2}} \leqslant K_{1}|\varepsilon| .
$$

We have $\bar{u}=\bar{v}+\bar{w} \in C(\bar{\Omega}) \cap H^{1}(\Omega), H \in H^{2}(\Omega) \cap C^{1}(\bar{\Omega})$ and $\beta \in H^{1}((0, \pi))$. Moreover, since $\mathcal{R} \in C^{1}(\bar{\Omega} \times \mathbb{R})$ and $z(\cdot ; \varepsilon) \in C \cap H^{1}$, then $\mathcal{R}_{*}(\cdot ; \varepsilon) \in C \cap H^{1}$ and $\left\|\mathcal{R}_{*}(\cdot ; \varepsilon)\right\|_{C}+\left\|\mathcal{R}_{*}(\cdot ; \varepsilon)\right\|_{H^{1}} \leqslant K_{2}$. Hence $f(t, x, \bar{u}(t, x ; \varepsilon) ; \varepsilon)=$ $\beta(x)(H(t, x)+\bar{u}(t, x ; \varepsilon))^{2 k}+\mathcal{R}_{*}(t, x ; \varepsilon) \in C \cap H^{1}$ and $\|f\|_{C}+\|f\|_{H^{1}} \leqslant K_{3}$.

Therefore, since $\bar{w}$ solves the range equation $\bar{w}=\varepsilon \square^{-1} \Pi_{N^{\perp}} f, \Pi_{N^{\perp}}$ satisfies (2.6), (2.7) and $\square^{-1}$ satisfies (2.3), we deduce that $\bar{w} \in C^{1}(\bar{\Omega}) \cap H^{2}(\Omega)$ and (4.52).

Step 2: $\bar{v}_{t} \in L^{\infty}(\Omega)$.

Let define

$$
\kappa_{18}:=k 4^{-k} \min _{\Omega_{1 / 8}}\left(\beta H^{2 k-1}\right)
$$

and $0<\varepsilon_{4} \leqslant \varepsilon_{3}$ such that, $\forall|\varepsilon| \leqslant \varepsilon_{4}$

$$
|\varepsilon|^{-1+1 /(2 k)}\left\|\partial_{u} \mathcal{R}(z)\right\|_{L^{\infty}(\Omega)} \leqslant \frac{\kappa_{18}}{2}, \quad 4 k\left|\beta(H+\bar{v}+\bar{w})^{2 k-1}-\beta(H+\bar{v})^{2 k-1}\right|_{L^{\infty}} \leqslant \pi \frac{\kappa_{18}}{2}
$$

(such $\varepsilon_{4}$ exists by (1.7) and since $\|w\|_{E}=\mathrm{O}(\varepsilon)$ ). We claim that

$$
\begin{gathered}
\int_{\Omega}\left[2 k \beta(H+\bar{u})^{2 k-1}+\varepsilon^{-1+1 /(2 k)} \partial_{u} \mathcal{R}(z)\right] v \varphi \geqslant\left(10 \pi \kappa_{18} M-\kappa_{19}\|\hat{v}\|_{L^{2}(\mathbb{T})}\right)\|q(\hat{v})\|_{L^{1}(\mathbb{T})} \\
\forall|\varepsilon| \leqslant \varepsilon_{4}, \quad \forall v=v_{+}-v_{-} \in N, v_{ \pm}(t, x)=\hat{v}(t \pm x), \varphi:=q\left(v_{+}\right)-q\left(v_{-}\right) \in N,
\end{gathered}
$$

where $q=q_{M}(M>0)$ was defined in (4.20). Noting that $\int_{\Omega} \beta \bar{v}^{2 k-1} v \varphi=0$ by (2.19), $v \varphi \geqslant 0$ and using (2.22),

$$
\begin{aligned}
\int_{\Omega} 2 k \beta(H+\bar{v})^{2 k-1} v \varphi & =\int_{\Omega} 2 k \beta\left((H+\bar{v})^{2 k-1}-\bar{v}^{2 k-1}\right) v \varphi \geqslant \int_{\Omega} 2 k 4^{1-k} \beta H^{2 k-1} v \varphi \\
& \geqslant 2 k 4^{1-k} \min _{\Omega_{1 / 8}}\left(\beta H^{2 k-1}\right) \int_{\Omega_{1 / 8}} v \varphi=8 \kappa_{18} \int_{\Omega_{1 / 8}} v \varphi .
\end{aligned}
$$

Using (2.11), (2.12) we obtain the lower bound

$$
\begin{aligned}
\int_{\Omega_{1 / 8}} v \varphi & \geqslant \int_{\Omega_{1 / 8}} v_{+} q_{+}+v_{-} q_{-}-\int_{\Omega}\left|v_{-}\right|\left|q_{+}\right|+\left|v_{+}\right|\left|q_{-}\right| \\
& \geqslant 2 \pi\left(1-2 \frac{1}{8}\right) \int_{0}^{2 \pi} \hat{v}(s) q(\hat{v}(s)) \mathrm{d} s-2 \int_{\Omega}|\hat{v}(t-x)||q(\hat{v}(t+x))|
\end{aligned}
$$

\footnotetext{
7 However, such $K_{i}$ can be taken independently of $\varepsilon$ if we assume the further hypothesis (1.9) on $\mathcal{R}$, see Remarks 1.3 and 4.10 .
} 


$$
\geqslant \frac{3 \pi}{2} \int_{0}^{2 \pi} \hat{v}(s) q(\hat{v}(s)) \mathrm{d} s-\sqrt{2 \pi}\|\hat{v}\|_{L^{2}(\mathbb{T})}\|q(\hat{v})\|_{L^{1}(\mathbb{T})},
$$

and, by (4.56), we get

$$
\int_{\Omega} 2 k \beta(H+\bar{v})^{2 k-1} v \varphi \geqslant 12 \pi \kappa_{18} \int_{0}^{2 \pi} \hat{v}(s) q(\hat{v}(s)) \mathrm{d} s-\kappa_{19}\|\hat{v}\|_{L^{2}(\mathbb{T})}\|q(\hat{v})\|_{L^{1}(\mathbb{T})} .
$$

Since $\int_{\Omega}|v \varphi|=\int_{\Omega} v \varphi=2 \pi \int_{0}^{2 \pi} \hat{v}(s) q(\hat{v}(s)) \mathrm{d} s$ and using (4.54), we get, $\forall|\varepsilon| \leqslant \varepsilon_{4}$,

$$
\left|\int_{\Omega} \varepsilon^{-1+1 /(2 k)} \partial_{u} \mathcal{R}(z) v \varphi\right| \leqslant|\varepsilon|^{-1+1 /(2 k)}\left\|\partial_{u} \mathcal{R}(z)\right\|_{L^{\infty}(\Omega)} \int_{\Omega} v \varphi \leqslant \pi \kappa_{18} \int_{0}^{2 \pi} \hat{v}(s) q(\hat{v}(s)) \mathrm{d} s .
$$

Therefore, using (4.57), (4.58), the second inequality in (4.54) and since $v \varphi \geqslant 0$, we obtain

$$
\begin{aligned}
\int_{\Omega} & {\left[2 k \beta(H+\bar{u})^{2 k-1}+\varepsilon^{-1+1 /(2 k)} \partial_{u} \mathcal{R}(z)\right] v \varphi } \\
= & \int_{\Omega} 2 k \beta(H+\bar{v})^{2 k-1} v \varphi+\varepsilon^{-1+1 /(2 k)} \partial_{u} \mathcal{R}(z) v \varphi+2 k \beta\left[(H+\bar{v}+\bar{w})^{2 k-1}-(H+\bar{v})^{2 k-1}\right] v \varphi \\
\geqslant & 10 \pi \kappa_{18} \int_{0}^{2 \pi} \hat{v}(s) q(\hat{v}(s)) \mathrm{d} s-\kappa_{19}\|\hat{v}\|_{L^{2}(\mathbb{T})}\|q(\hat{v})\|_{L^{1}(\mathbb{T})}, \quad \forall|\varepsilon| \leqslant \varepsilon_{4} .
\end{aligned}
$$

Since $\lambda q(\lambda) \geqslant M|q(\lambda)|$

$$
\int_{0}^{2 \pi} \hat{v}(s) q(\hat{v}(s)) \mathrm{d} s \geqslant M \int_{0}^{2 \pi}|q(\hat{v}(s))| \mathrm{d} s=M\|q(\hat{v})\|_{L^{1}(\mathbb{T})},
$$

and, by (4.59), we finally get (4.55). We now conclude the proof that $\bar{v}_{t} \in L^{\infty}(\Omega)$. Taking $v:=\bar{v}_{t}$ in (4.55) and $\varphi:=q\left(\partial_{t} \bar{v}_{+}\right)-q\left(\partial_{t} \bar{v}_{-}\right)$we obtain ${ }^{8}$ (recall that $\left|\hat{\bar{v}}^{\prime}\right|_{L^{2}(\mathbb{T})} \leqslant R_{*}$ which is fixed)

$$
\int_{\Omega}\left[2 k \beta(H+\bar{u})^{2 k-1}+\varepsilon^{-1+1 /(2 k)} \partial_{u} \mathcal{R}(z)\right] \bar{v}_{t} \varphi \geqslant\left(10 \pi \kappa_{18} M-\kappa_{20}\right)\left\|q\left(\hat{\bar{v}}^{\prime}\right)\right\|_{L^{1}(\mathbb{T})}, \quad \forall|\varepsilon| \leqslant \varepsilon_{4} .
$$

Note that, since $H, \bar{w} \in C^{1}$, by (1.7) ( $A$ is defined in (4.50))

$$
\|A\|_{L^{\infty}} \leqslant K_{4} .
$$

From (4.51), (4.52), (4.61), we get

$$
\left|\int_{\Omega}\left[2 k \beta(H+\bar{u})^{2 k-1}+\varepsilon^{-1+1 /(2 k)} \partial_{u} \mathcal{R}(z)\right] \bar{v}_{t} \varphi\right|=\left|\int_{\Omega}\left[2 k \beta(H+\bar{u})^{2 k-1}\left(H_{t}+\bar{w}_{t}\right)+A\right] \varphi\right| \leqslant K_{5}\|\varphi\|_{L^{1}(\Omega)}
$$

and, by (4.60), we deduce

$$
\left(10 \pi \kappa_{18} M-\kappa_{20}\right)\left\|q\left(\hat{\bar{v}}^{\prime}\right)\right\|_{L^{1}(\mathbb{T})} \leqslant K_{5}\|\varphi\|_{L^{1}(\Omega)}, \quad \forall|\varepsilon| \leqslant \varepsilon_{4} .
$$

Finally, recalling $\|\varphi\|_{L^{1}} \leqslant 2 \pi\left\|q\left(\hat{\bar{v}}^{\prime}\right)\right\|_{L^{1}(\mathbb{T})}$ (see (4.31)), we find

$$
M\left\|q\left(\hat{\bar{v}}^{\prime}\right)\right\|_{L^{1}(\mathbb{T})} \leqslant K_{6}\left\|q\left(\hat{\bar{v}}^{\prime}\right)\right\|_{L^{1}(\mathbb{T})} .
$$

\footnotetext{
${ }^{8}$ Here $\bar{v}(t, x)=\hat{\bar{v}}(t+x)-\hat{\bar{v}}(t-x)$ and so $\bar{v}_{t}(t, x)=\hat{\bar{v}}^{\prime}(t+x)-\hat{\bar{v}}^{\prime}(t-x)$.
} 
We claim that (4.62) implies $\hat{\bar{v}}^{\prime} \in L^{\infty}(\mathbb{T})$. Indeed, if $\hat{\bar{v}}^{\prime} \notin L^{\infty}(\mathbb{T})$, then for any $M>0,\left\|q\left(\hat{\bar{v}}^{\prime}\right)\right\|_{L^{1}(\mathbb{T})}>0$ and (4.62) yields $M \leqslant K_{6}$; hence $\hat{\bar{v}}^{\prime} \in L^{\infty}(\mathbb{T})$. Taking $M:=\left\|\hat{\bar{v}}^{\prime}\right\|_{L^{\infty}(\mathbb{T})} / 2$ we obtain, by (4.62), $\left\|\hat{\bar{v}}^{\prime}\right\|_{L^{\infty}(\mathbb{T})} \leqslant 2 K_{6}$ and $\left\|\bar{v}_{t}\right\|_{L^{\infty}(\Omega)} \leqslant 4 K_{6}$ by $(2.15)$.

Step 3: $\bar{v}_{t} \in H^{1}(\Omega)$ (and hence $\bar{v} \in N \cap C^{1}(\Omega) \cap H^{2}(\Omega)$ ).

We claim that

$$
\int_{\Omega}\left[2 k \beta(H+\bar{u})^{2 k-1}+\varepsilon^{-1+1 /(2 k)}\left(\partial_{u} \mathcal{R}(z)\right)\right] v^{2} \geqslant \kappa_{18}\|v\|_{L^{2}}^{2}, \quad \forall v \in N,|\varepsilon| \leqslant \varepsilon_{4},
$$

where $\kappa_{18}$ is defined in (4.53) and $\varepsilon_{4}$ is defined in (4.54). Arguing as before, using $\int_{\Omega} \beta \bar{v}^{2 k-1} v^{2}=0$ (recall (2.19)), (2.22) and (4.16), (4.15)

$$
\begin{aligned}
\int_{\Omega} 2 k \beta(H+\bar{v})^{2 k-1} v^{2} & =\int_{\Omega} 2 k \beta\left((H+\bar{v})^{2 k-1}-\bar{v}^{2 k-1}\right) v^{2} \geqslant \int_{\Omega} 2 k 4^{1-k} \beta H^{2 k-1} v^{2} \\
& \geqslant 2 k 4^{1-k} c_{1}\left(\beta H^{2 k-1}\right) \int_{\Omega} v^{2}=2 \kappa_{18}\|v\|_{L^{2}}^{2} .
\end{aligned}
$$

Since, by (4.54),

$$
\left|\int_{\Omega} \varepsilon^{-1+1 /(2 k)}\left(\partial_{u} \mathcal{R}(z)\right) v^{2}\right| \leqslant|\varepsilon|^{-1+1 /(2 k)}\left\|\partial_{u} \mathcal{R}(z)\right\|_{L^{\infty}}\|v\|_{L^{2}}^{2} \leqslant \frac{\kappa_{18}}{2}\|v\|_{L^{2}}^{2}
$$

and

$$
\left|\int_{\Omega} 2 k \beta\left[(H+\bar{u})^{2 k-1}-(H+\bar{v})^{2 k-1}\right] v^{2}\right| \leqslant\left|2 k \beta\left[(H+\bar{u})^{2 k-1}-(H+\bar{v})^{2 k-1}\right]\right|_{L^{\infty}}\|v\|_{L^{2}}^{2} \leqslant \frac{\kappa_{18}}{2}\|v\|_{L^{2}}^{2}
$$

using (4.64) we prove (4.63).

Take $\varphi=-D_{-h} D_{h} \bar{v}_{t} \in N$ in (4.51). Integrating by part (recall (2.26) and (2.23)) equality (4.51), we obtain

$$
\begin{aligned}
0= & \int_{\Omega} D_{h}\left[2 k \beta(H+\bar{u})^{2 k-1}\left(H_{t}+\bar{v}_{t}+\bar{w}_{t}\right)+\varepsilon^{-1+1 /(2 k)} \partial_{u} \mathcal{R}(z) \bar{v}_{t}+A\right] D_{h} \bar{v}_{t} \\
= & \int_{\Omega}\left[2 k \beta(H+\bar{u})^{2 k-1}+\varepsilon^{-1+1 /(2 k)}\left(\partial_{u} \mathcal{R}(z)\right)\right]\left(D_{h} \bar{v}_{t}\right)^{2} \\
& +\left[2 k \beta\left(D_{h}\left((H+\bar{u})^{2 k-1}\right)\right)\left(T_{h} \bar{v}_{t}\right)+2 k \beta\left(D_{h}\left((H+\bar{u})^{2 k-1}\left(H_{t}+\bar{w}_{t}\right)\right)\right)\right. \\
& \left.+\varepsilon^{-1+1 /(2 k)}\left(D_{h}\left(\partial_{u} \mathcal{R}(z)\right)\right)\left(T_{h} \bar{v}_{t}\right)+D_{h} A\right] D_{h} \bar{v}_{t} .
\end{aligned}
$$

The dominant term here is (4.65). Using (4.63) with $v:=D_{h} \bar{v}_{t}$, we estimate (4.65) by

$$
\int_{\Omega}\left[2 k \beta(H+\bar{u})^{2 k-1}+\varepsilon^{-1+1 /(2 k)}\left(\partial_{u} \mathcal{R}(z)\right)\right]\left(D_{h} \bar{v}_{t}\right)^{2} \geqslant \kappa_{18}\left\|D_{h} \bar{v}_{t}\right\|_{L^{2}}^{2}, \quad \forall|\varepsilon| \leqslant \varepsilon_{4} .
$$

We now estimate all the other terms. Since $\left\|T_{h} \bar{v}_{t}\right\|_{L^{\infty}(\Omega)}=\left\|\bar{v}_{t}\right\|_{L^{\infty}(\Omega)} \leqslant K_{7}$ and

$$
\left\|D_{h}\left((H+\bar{u})^{2 k-1}\right)\right\|_{L^{2}} \leqslant\left\|\partial_{t}\left((H+\bar{u})^{2 k-1}\right)\right\|_{L^{2}}=(2 k-1)\left\|(H+\bar{u})^{2 k-2}\left(H_{t}+\bar{u}_{t}\right)\right\|_{L^{2}} \leqslant \kappa_{21},
$$

we obtain

$$
\left|\int_{\Omega}\left[2 k \beta\left(D_{h}\left((H+\bar{u})^{2 k-1}\right)\right)\left(T_{h} \bar{v}_{t}\right)\right] D_{h} \bar{v}_{t}\right| \leqslant K_{8}\left\|D_{h} \bar{v}_{t}\right\|_{L^{2}} .
$$

Since $H \in C^{1} \cap H^{2}, \bar{v}_{t} \in L^{\infty}, \bar{w} \in C^{1} \cap H^{2}$, we have

$$
\begin{aligned}
& \left\|D_{h}\left((H+\bar{u})^{2 k-1}\left(H_{t}+\bar{w}_{t}\right)\right)\right\|_{L^{2}} \leqslant\left\|\partial_{t}\left((H+\bar{u})^{2 k-1}\left(H_{t}+\bar{w}_{t}\right)\right)\right\|_{L^{2}} \\
& \quad=\left\|(2 k-1)(H+\bar{u})^{2 k-2}\left(H_{t}+\bar{u}_{t}\right)\left(H_{t}+\bar{w}_{t}\right)+(H+\bar{u})^{2 k-1}\left(H_{t t}+\bar{w}_{t t}\right)\right\|_{L^{2}} \leqslant K_{9},
\end{aligned}
$$


and we deduce

$$
\left|\int_{\Omega}\left[2 k \beta\left(D_{h}\left((H+\bar{u})^{2 k-1}\left(H_{t}+\bar{w}_{t}\right)\right)\right)\right] D_{h} \bar{v}_{t}\right| \leqslant K_{9}\left\|D_{h} \bar{v}_{t}\right\|_{L^{2}}
$$

From

$$
\varepsilon^{-1+1 /(2 k)} \partial_{t}\left(\partial_{u} \mathcal{R}(z)\right)=\varepsilon^{-1+1 /(2 k)} \partial_{t u}^{2} \mathcal{R}(z)+\varepsilon^{-1+1 / k} \partial_{u u}^{2} \mathcal{R}(z)\left(H_{t}+\bar{u}_{t}\right)
$$

we derive

$$
\left\|\varepsilon^{-1+1 /(2 k)} D_{h}\left(\partial_{u} \mathcal{R}(z)\right)\right\|_{L^{2}} \leqslant\left\|\varepsilon^{-1+1 /(2 k)} \partial_{t}\left(\partial_{u} \mathcal{R}(z)\right)\right\|_{L^{2}} \leqslant K_{10}
$$

and (recall $\bar{v}_{t} \in L^{\infty}$ )

$$
\left|\int_{\Omega}\left[\varepsilon^{-1+1 /(2 k)}\left(D_{h}\left(\partial_{u} \mathcal{R}(z)\right)\right) \bar{v}_{t}\right] D_{h} \bar{v}_{t}\right| \leqslant K_{10}\left\|\bar{v}_{t}\right\|_{L^{\infty}}\left\|D_{h} \bar{v}_{t}\right\|_{L^{2}}=K_{11}\left\|D_{h} \bar{v}_{t}\right\|_{L^{2}} .
$$

We finally estimate the term

$$
\left|\int_{\Omega}\left(D_{h} A\right)\left(D_{h} \bar{v}_{t}\right)\right| \leqslant\left\|D_{h} A\right\|_{L^{2}}\left\|D_{h} \bar{v}_{t}\right\|_{L^{2}} \stackrel{(2.28)}{\leqslant}\left\|\partial_{t} A\right\|_{L^{2}}\left\|D_{h} \bar{v}_{t}\right\|_{L^{2}}
$$

( $A$ is defined in (4.50)). Since

$$
\begin{aligned}
\partial_{t} A= & \varepsilon^{-1} \partial_{t t}^{2} \mathcal{R}(z)+\varepsilon^{-1+1 /(2 k)} \partial_{t u}^{2} \mathcal{R}(z)\left(2 H_{t}+\bar{v}_{t}+2 \bar{w}_{t}\right) \\
& +\varepsilon^{-1+1 / k} \partial_{u u}^{2} \mathcal{R}(z)\left(H_{t}+\bar{u}_{t}\right)\left(H_{t}+\bar{w}_{t}\right)+\varepsilon^{-1+1 /(2 k)} \partial_{u} \mathcal{R}(z)\left(H_{t t}+\bar{w}_{t t}\right),
\end{aligned}
$$

by (1.7), and using that $\bar{v}_{t} \in L^{\infty}(\Omega), \bar{w}, H \in H^{2}(\Omega) \cap C^{1}(\Omega)$, then $\left\|\partial_{t} A\right\|_{L^{2}} \leqslant K_{12}$ and

$$
\left|\int_{\Omega}\left(D_{h} A\right)\left(D_{h} \bar{v}_{t}\right)\right| \leqslant K_{12}\left\|D_{h} \bar{v}_{t}\right\|_{L^{2}}
$$

Recollecting (4.65), (4.66), (4.68), (4.69), (4.71) and (4.72) we obtain

$$
\kappa_{18}\left\|D_{h} \bar{v}_{t}\right\|_{L^{2}}^{2} \leqslant K_{13}\left\|D_{h} \bar{v}_{t}\right\|_{L^{2}(\Omega)}
$$

and so $\left\|D_{h} \bar{v}_{t}\right\|_{L^{2}(\Omega)} \leqslant K_{14}$ for all $h$. By (2.27) we conclude that $\bar{v}_{t} \in N \cap H^{1}$ and $\left\|\bar{v}_{t t}\right\|_{L^{2}(\Omega)} \leqslant K_{14}$.

We now prove Theorem 2(ii) by induction over $j \geqslant 1$.

Lemma 4.9. Assuming $\bar{v}, \bar{w} \in C^{j-1}(\bar{\Omega}) \cap H^{j}(\Omega)$, then $\bar{v}, \bar{w} \in C^{j}(\bar{\Omega}) \cap H^{j+1}(\Omega)$.

Proof. Again we divide the proof in three steps.

Step 1: $\bar{w} \in C^{j}(\bar{\Omega}) \cap H^{j+1}(\Omega)$ and

$$
\|\bar{w}\|_{C^{j}}+\|\bar{w}\|_{H^{j+1}} \leqslant K_{1}^{(j)}|\varepsilon| .
$$

By hypotheses $H \in C^{j}(\bar{\Omega}) \cap H^{j+1}(\Omega)$ (since $\left.h \in C^{j-1}(\bar{\Omega}) \cap H^{j}(\Omega)\right), \beta \in H^{j}((0, \pi)), \mathcal{R} \in C^{j}(\bar{\Omega} \times \mathbb{R})$ and $z(\cdot ; \varepsilon) \in C^{j-1}(\bar{\Omega}) \cap H^{j}(\Omega)$. Hence $\mathcal{R}_{*}(\cdot ; \varepsilon) \in C^{j-1}(\bar{\Omega}) \cap H^{j}(\Omega)$ and $\left\|\mathcal{R}_{*}(\cdot ; \varepsilon)\right\|_{C^{j-1}}+\left\|\mathcal{R}_{*}(\cdot ; \varepsilon)\right\|_{H^{j}} \leqslant K_{2}^{(j)}$. Hence $f(t, x, \bar{u}(t, x ; \varepsilon) ; \varepsilon)=\beta(x)(H(t, x)+\bar{u}(t, x ; \varepsilon))^{2 k}+\mathcal{R}_{*}(t, x ; \varepsilon) \in C^{j-1}(\bar{\Omega}) \cap H^{j}(\Omega)$ and $\|f\|_{C^{j-1}}+$ $\|f\|_{H^{j}} \leqslant K_{3}^{(j)}$.

Since $\bar{w}$ solves the range equation $\bar{w}=\varepsilon \square^{-1} \Pi_{N^{\perp}} f, \Pi_{N^{\perp}}$ satisfies (2.6), (2.7) and $\square^{-1}$ satisfies (2.3), we conclude that $\bar{w} \in C^{j}(\bar{\Omega}) \cap H^{j+1}(\Omega)$ and that (4.73) holds.

Step 2: $\partial_{t}^{j} \bar{v} \in L^{\infty}(\Omega)$.

Reasoning as for (4.49), we get

$$
0=\int_{\Omega} \partial_{t}^{j}\left(\beta(H+\bar{v}+\bar{w})^{2 k}+\mathcal{R}_{*}\right) \varphi=\int_{\Omega}\left[2 k \beta(H+\bar{u})^{2 k-1}+\varepsilon^{-1+1 /(2 k)} \partial_{u} \mathcal{R}(z)\right]\left(\partial_{t}^{j} \bar{v}\right) \varphi+\mathcal{F}^{(j)} \varphi
$$


for any $\varphi \in N$. Here $\mathcal{F}^{(j)}$ depends polynomially on $k, \beta$ and

$$
\varepsilon^{-1+n /(2 k)} \partial_{t}^{l} \partial_{u}^{n} \mathcal{R}(z), \quad l+n=j, \quad \partial_{t}^{l} H, \partial_{t}^{l} \bar{w}, \quad l \leqslant j, \quad \partial_{t}^{l} \bar{v}, \quad l \leqslant j-1,
$$

but not on $\partial_{t}^{j} \bar{v}$.

Choosing in (4.55) $v=\partial_{t}^{j} \bar{v}$ and so $\varphi:=q\left(\partial_{t}^{j} \bar{v}_{+}\right)-q\left(\partial_{t}^{j} \bar{v}_{-}\right)$, we get (recall that $\left.\bar{v} \in H^{j}\right), \forall|\varepsilon| \leqslant \varepsilon_{4}$,

$$
\int_{\Omega}\left[2 k \beta(H+\bar{u})^{2 k-1}+\varepsilon^{-1+1 /(2 k)} \partial_{u} \mathcal{R}(z)\right]\left(\partial_{t}^{j} \bar{v}\right) \varphi \geqslant\left(10 \pi \kappa_{18} M-\kappa_{24}\right)\left\|q\left(\hat{\bar{v}}^{(j)}\right)\right\|_{L^{1}(\mathbb{T})},
$$

where $\hat{\bar{v}}^{(j)}(\xi):=\frac{\mathrm{d}^{j}}{\mathrm{~d} \xi^{j}} \hat{\bar{v}}$. On the other hand, by (4.74) we get

$$
\int_{\Omega}\left[2 k \beta(H+\bar{u})^{2 k-1}+\varepsilon^{-1+1 /(2 k)} \partial_{u} \mathcal{R}(z)\right]\left(\partial_{t}^{j} \bar{v}\right) \varphi \leqslant\left\|\mathcal{F}^{(j)}\right\|_{L^{\infty}}\|\varphi\|_{L^{1}} \leqslant K_{4}^{(j)}\|\varphi\|_{L^{1}} .
$$

Since $\|\varphi\|_{L^{1}} \leqslant 2 \pi\left\|q\left(\hat{\bar{v}}^{(j)}\right)\right\|_{L^{1}(\mathbb{T})}$ (see (4.31)), from (4.75) and (4.76) we get

$$
\left(10 \pi \kappa_{18} M-\kappa_{24}\right)\left\|q\left(\hat{\bar{v}}^{(j)}\right)\right\|_{L^{1}(\mathbb{T})} \leqslant K_{5}^{(j)}\left\|q\left(\hat{\bar{v}}^{(j)}\right)\right\|_{L^{1}(\mathbb{T})}, \quad \forall|\varepsilon| \leqslant \varepsilon_{4} .
$$

Then

$$
M\left\|q\left(\hat{\bar{v}}^{(j)}\right)\right\|_{L^{1}(\mathbb{T})} \leqslant K_{6}^{(j)}\left\|q\left(\hat{\bar{v}}^{(j)}\right)\right\|_{L^{1}(\mathbb{T})}, \quad \forall|\varepsilon| \leqslant \varepsilon_{4} .
$$

Arguing as in (4.62) we get $\hat{\bar{v}}^{(j)} \in L^{\infty}(\mathbb{T})$. Finally $\partial_{t}^{j} \bar{v} \in L^{\infty}(\Omega)$ and $\left\|\partial_{t}^{j} \bar{v}\right\|_{L^{\infty}(\Omega)} \leqslant 4 K_{6}^{(j)}$.

Step 3: We now prove that $\partial_{t}^{j} \bar{v} \in H^{1}$ (and hence $\bar{v} \in N \cap C^{j} \cap H^{j+1}$ ).

Choosing $\varphi:=-D_{-h} D_{h} \partial_{t}^{j} \bar{v}$ in (4.74), integrating by parts (recall (2.26) and (2.23))

$$
\begin{aligned}
0= & \int_{\Omega}\left[2 k \beta(H+\bar{u})^{2 k-1}+\varepsilon^{-1+1 /(2 k)} \partial_{u} \mathcal{R}(z)\right]\left(D_{h}\left(\partial_{t}^{j} \bar{v}\right)\right)^{2} \\
& +\left[\left(D_{h}\left(2 k \beta(H+\bar{u})^{2 k-1}+\varepsilon^{-1+1 /(2 k)} \partial_{u} \mathcal{R}(z)\right)\right)\left(T_{h}\left(\partial_{t}^{j} \bar{v}\right)\right)+\left(D_{h}\left(\mathcal{F}^{(j)}\right)\right)\right]\left(D_{h}\left(\partial_{t}^{j} \bar{v}\right)\right) .
\end{aligned}
$$

Using (4.63) we get

$$
\int_{\Omega}\left[2 k \beta(H+\bar{u})^{2 k-1}+\varepsilon^{-1+1 /(2 k)} \partial_{u} \mathcal{R}(z)\right]\left(D_{h}\left(\partial_{t}^{j} \bar{v}\right)\right)^{2} \geqslant \kappa_{18}\left\|D_{h}\left(\partial_{t}^{j} \bar{v}\right)\right\|_{L^{2}}^{2}, \quad \forall|\varepsilon| \leqslant \varepsilon_{4} .
$$

From (4.67), (4.70) and since $\partial_{t}^{j} \bar{v} \in L^{\infty}(\Omega)$,

$$
\begin{aligned}
& \left|\int_{\Omega}\left(D_{h}\left(2 k \beta(H+\bar{u})^{2 k-1}+\varepsilon^{-1+1 /(2 k)} \partial_{u} \mathcal{R}(z)\right)\right)\left(T_{h}\left(\partial_{t}^{j} \bar{v}\right)\right)\left(D_{h}\left(\partial_{t}^{j} \bar{v}\right)\right)\right| \\
& \quad \leqslant 4 K_{6}^{(j)}\left\|D_{h}\left(2 k \beta(H+\bar{u})^{2 k-1}+\varepsilon^{-1+1 /(2 k)} \partial_{u} \mathcal{R}(z)\right)\right\|_{L^{2}}\left\|D_{h}\left(\partial_{t}^{j} \bar{v}\right)\right\|_{L^{2}} \\
& \quad \leqslant K_{7}^{(j)}\left\|D_{h}\left(\partial_{t}^{j} \bar{v}\right)\right\|_{L^{2}} .
\end{aligned}
$$

We note that $\partial_{t} \mathcal{F}^{(j)}$ is a polynomial in $k, \beta$,

$$
\varepsilon^{-1+n /(2 k)} \partial_{t}^{l} \partial_{u}^{n} \mathcal{R}(z), \quad l+n=j+1, \quad \partial_{t}^{l} H, \partial_{t}^{l} \bar{w}, \quad l \leqslant j+1, \quad \partial_{t}^{l} \bar{v}, \quad l \leqslant j,
$$

and that the terms $\partial_{t}^{j+1} H, \partial_{t}^{j+1} \bar{w} \in L^{2}(\Omega)$ (recall that $\bar{w}, H \in H^{j+1}(\Omega)$ by Step 1) appear only linearly (with no powers). Hence, using that $\partial_{t}^{l} H, \partial_{t}^{l} \bar{w}, \partial_{t}^{l} \bar{v} \in L^{\infty}(\Omega), \forall l \leqslant j$,

$$
\begin{aligned}
\left|\int_{\Omega}\left(D_{h}\left(\mathcal{F}^{(j)}\right)\right)\left(D_{h}\left(\partial_{t}^{j} \bar{v}\right)\right)\right| & \leqslant\left\|D_{h}\left(\mathcal{F}^{(j)}\right)\right\|_{L^{2}}\left\|D_{h}\left(\partial_{t}^{j} \bar{v}\right)\right\|_{L^{2}} \stackrel{(2.28)}{\leqslant}\left\|\partial_{t}\left(\mathcal{F}^{(j)}\right)\right\|_{L^{2}}\left\|D_{h}\left(\partial_{t}^{j} \bar{v}\right)\right\|_{L^{2}} \\
& \leqslant K_{8}^{(j)}\left\|D_{h}\left(\partial_{t}^{j} \bar{v}\right)\right\|_{L^{2}} .
\end{aligned}
$$


Finally, by (4.77)-(4.80) we get

$$
\kappa_{18}\left\|D_{h}\left(\partial_{t}^{j} \bar{v}\right)\right\|_{L^{2}}^{2} \leqslant K_{9}^{(j)}\left\|D_{h}\left(\partial_{t}^{j} \bar{v}\right)\right\|_{L^{2}},
$$

and therefore

$$
\left\|D_{h}\left(\partial_{t}^{j} \bar{v}\right)\right\|_{L^{2}} \leqslant K_{10}^{(j)}
$$

By (2.27), we conclude the proof obtaining $\partial_{t}^{j+1} \bar{v} \in L^{2}(\Omega)$ and $\left\|\partial_{t}^{j+1} \bar{v}\right\|_{L^{2}} \leqslant K_{10}^{(j)}$.

Remark 4.10. If $H, \bar{u} \in H^{i}(\Omega) \cap C^{i-1}(\bar{\Omega})(0 \leqslant i \leqslant j)$ and (1.9) holds, then $R_{*}$ and $\partial_{t} R_{*}$ are bounded in $H^{i}(\Omega) \cap C^{i-1}(\bar{\Omega})$ by some constant $\kappa_{i}$ independent of $\varepsilon$. In this case, the constants $K_{i}$ of this section can be taken independently of $\varepsilon$, obtaining the estimates (1.10).

\subsection{Proof of Theorem 1}

The following proposition is a sort of "maximum principle" for the wave equations (1.1)-(1.3).

Proposition 4.11. Let $h \in N^{\perp}, h>0$ (or $\left.h \geqslant 0\right)$ a.e. in $\Omega$. Then there exists a weak solution $H \in E$ of $\square H=h$ satisfying $H>0$ (or $H \geqslant 0$ ). In particular we can choose

$$
H(t, x):=\frac{1}{2} \int_{0}^{\kappa} \int_{t-x-\xi}^{t-x+\xi} h(\tau, \xi) \mathrm{d} \tau \mathrm{d} \xi-\frac{1}{2} \int_{\kappa}^{x} \int_{t-x+\xi}^{t+x-\xi} h(\tau, \xi) \mathrm{d} \tau \mathrm{d} \xi
$$

for a suitable $\kappa \in(0, \pi)$. Moreover $h \in C^{j-1} \Rightarrow H \in C^{j}$ and $h \in H^{j} \Rightarrow H \in H^{j+1}$, for $j \geqslant 1$.

Proof. We consider the case $h>0$, the case $h \geqslant 0$ being similar.

Step 1: $H$ defined in (4.81) belongs to $H^{1}(\Omega) \cap C^{1 / 2}(\bar{\Omega})$ for any $\kappa \in(0, \pi)$ and

$$
\begin{aligned}
2\left(\partial_{t} H\right)(t, x)= & \int_{0}^{\kappa}(h(t-x+\xi, \xi)-h(t-x-\xi, \xi)) \mathrm{d} \xi \\
& -\int_{\kappa}^{x}(h(t+x-\xi, \xi)-h(t-x+\xi, \xi)) \mathrm{d} \xi \in L^{2}(\Omega), \\
2\left(\partial_{x} H\right)(t, x)= & \int_{0}^{\kappa}(-h(t-x+\xi, \xi)+h(t-x-\xi, \xi)) \mathrm{d} \xi \\
& -\int_{\kappa}^{x}(h(t+x-\xi, \xi)+h(t-x+\xi, \xi)) \mathrm{d} \xi \in L^{2}(\Omega) .
\end{aligned}
$$

We shall prove that the first addendum in the r.h.s. of (4.81)

$$
H_{1}(t, x):=\frac{1}{2} \int_{0}^{\kappa} \int_{t-x-\xi}^{t-x+\xi} h(\tau, \xi) \mathrm{d} \tau \mathrm{d} \xi
$$

belongs to $C^{1 / 2}(\bar{\Omega}) \cap H^{1}(\Omega)$, the second addendum being analogous. Defining

$$
T(t, x):=T(t, x ; \kappa):=\{(\tau, \xi) \in \Omega \mid t-x-\xi<\tau<t-x+\xi, 0<\xi<\kappa\}
$$

we can write $H_{1}(t, x):=(1 / 2) \int_{T(t, x)} h(\tau, \xi) \mathrm{d} \tau \mathrm{d} \xi$. 
Since meas $(T(t, x ; \kappa))=\kappa^{2} \leqslant \pi^{2}$ we derive that $H_{1}$ is uniformly bounded by

$$
\left|H_{1}(t, x)\right| \leqslant \frac{1}{2} \int_{\Omega} \mathbf{1}_{T(t, x)}(\tau, \xi)|h(\tau, \xi)| \mathrm{d} \tau \mathrm{d} \xi \leqslant \frac{\pi}{2}\|h\|_{L^{2}(\Omega)}
$$

using Cauchy-Schwartz inequality.

For $i=1,2$ and $\left(t_{i}, x_{i}\right) \in \Omega$, let define $T_{i}:=T\left(t_{i}, x_{i}\right)$. It results

$$
\operatorname{meas}\left(T_{1} \backslash T_{2}\right)=\operatorname{meas}\left(T_{2} \backslash T_{1}\right) \leqslant \pi\left(\left|t_{1}-t_{2}\right|+\left|x_{1}-x_{2}\right|\right)
$$

and, using again Cauchy-Schwartz inequality,

$$
\left|H_{1}\left(t_{1}, x_{1}\right)-H_{1}\left(t_{2}, x_{2}\right)\right| \leqslant \frac{1}{2} \int_{T_{1} \backslash T_{2}}|h|+\frac{1}{2} \int_{T_{2} \backslash T_{1}}|h| \leqslant \sqrt{\pi}\left(\left|t_{1}-t_{2}\right|+\left|x_{1}-x_{2}\right|\right)^{1 / 2}\|h\|_{L^{2}(\Omega)} .
$$

Therefore we have proved $H_{1} \in C^{1 / 2}(\bar{\Omega})$.

We now prove that $H_{1} \in H^{1}(\Omega)$ and that $\partial_{t} H_{1}=-\partial_{x} H_{1}=f_{1}$ where

$$
f_{1}(t, x):=\frac{1}{2} \int_{0}^{\kappa}(h(t-x+\xi, \xi)-h(t-x-\xi, \xi)) \mathrm{d} \xi \in L^{2}(\Omega) .
$$

We first justify that $f_{1} \in L^{2}(\Omega)$. Since

$$
|h(t-x+\xi, \xi)|^{2}+|h(t-x-\xi, \xi)|^{2} \geqslant \frac{1}{2}|h(t-x+\xi, \xi)-h(t-x-\xi, \xi)|^{2},
$$

by periodicity w.r.t. $t$ we obtain that, $\forall x \in(0, \pi)$,

$$
\|h\|_{L^{2}(\Omega)}^{2} \geqslant \int_{0}^{\kappa} \int_{0}^{2 \pi}|h(t, \xi)|^{2} \mathrm{~d} t \mathrm{~d} \xi \geqslant \frac{1}{4} \int_{0}^{\kappa} \int_{0}^{2 \pi}|h(t-x+\xi, \xi)-h(t-x-\xi, \xi)|^{2} \mathrm{~d} t \mathrm{~d} \xi .
$$

Integrating the previous inequality in the variable $x$ between 0 and $\pi$, applying Fubini Theorem and Cauchy-Schwartz inequality, we deduce

$$
\begin{aligned}
\pi\|h\|_{L^{2}(\Omega)}^{2} & \geqslant \frac{1}{4} \int_{0}^{\pi} \int_{0}^{\kappa} \int_{0}^{2 \pi}|h(t-x+\xi, \xi)-h(t-x-\xi, \xi)|^{2} \mathrm{~d} t \mathrm{~d} \xi \mathrm{d} x \\
& =\frac{1}{4} \int_{\Omega} \int_{0}^{\kappa}|h(t-x+\xi, \xi)-h(t-x-\xi, \xi)|^{2} \mathrm{~d} \xi \mathrm{d} t \mathrm{~d} x \\
& \geqslant \frac{1}{4 \kappa} \int_{\Omega}\left(\int_{0}^{\kappa}|h(t-x+\xi, \xi)-h(t-x-\xi, \xi)| \mathrm{d} \xi\right)^{2} \mathrm{~d} t \mathrm{~d} x \\
& \geqslant \frac{1}{\kappa} \int_{\Omega} f_{1}^{2}(t, x) \mathrm{d} t \mathrm{~d} x=\frac{1}{\kappa}\left\|f_{1}\right\|_{L^{2}}^{2} .
\end{aligned}
$$

Finally, we prove that $\partial_{x} H_{1}=-f_{1}$, being $\partial_{t} H_{1}=f_{1}$ analogous. By Fubini Theorem

$$
\begin{aligned}
2 \int_{\Omega} H_{1} \varphi_{x} & =\int_{0}^{2 \pi} \int_{0}^{\kappa} \int_{0}^{\pi} \int_{t-x-\xi}^{t-x+\xi} h(\tau, \xi) \varphi_{x}(t, x) \mathrm{d} \tau \mathrm{d} x \mathrm{~d} \xi \mathrm{d} t \\
& =\int_{0}^{2 \pi} \int_{0}^{\kappa}\left(\int_{t+\xi-\pi}^{t+\xi} h \int_{0}^{t+\xi-\tau} \varphi_{x} \mathrm{~d} x \mathrm{~d} \tau+\int_{t-\xi}^{t+\xi-\pi} h \int_{0}^{\pi} \varphi_{x} \mathrm{~d} x \mathrm{~d} \tau+\int_{t-\xi-\pi}^{t-\xi} h \int_{t-\xi-\tau}^{\pi} \varphi_{x} \mathrm{~d} x \mathrm{~d} \tau\right) \mathrm{d} \xi \mathrm{d} t
\end{aligned}
$$




$$
\begin{aligned}
& =\int_{0}^{2 \pi} \int_{0}^{\kappa}\left(\int_{t+\xi-\pi}^{t+\xi} h(\tau, \xi) \varphi(t, t+\xi-\tau) \mathrm{d} \tau-\int_{t-\xi-\pi}^{t-\xi} h(\tau, \xi) \varphi(t, t-\xi-\tau) \mathrm{d} \tau\right) \mathrm{d} \xi \mathrm{d} t \\
& =\int_{0}^{2 \pi} \int_{0}^{\kappa}\left(-\int_{\pi}^{0} h(t+\xi-x, \xi) \varphi(t, x) \mathrm{d} x+\int_{\pi}^{0} h(t-\xi-x, \xi) \varphi(t, x) \mathrm{d} x\right) \mathrm{d} \xi \mathrm{d} t=2 \int_{\Omega} f_{1} \varphi .
\end{aligned}
$$

With analogue computations for the second addendum of $H$ in (4.81) we derive (4.82) and (4.83).

Step 2: There exists $\kappa \in(0, \pi)$ such that $H(t, x)$ verifies the Dirichlet boundary conditions $H(t, 0)=H(t, \pi)=0$ $\forall t \in \mathbb{T}$.

By (4.81), the function $H$ satisfies, for any $\kappa \in(0, \pi), H(t, 0)=0 \forall t \in \mathbb{T}$. It remains to find $\kappa$ imposing $H(t, \pi)=0$. Taking $x=\pi$ in (4.81) we obtain

$$
\begin{aligned}
H(t, \pi) & :=\frac{1}{2} \int_{0}^{\kappa} \int_{t-\pi-\xi}^{t-\pi+\xi} h(\tau, \xi) \mathrm{d} \tau \mathrm{d} \xi-\frac{1}{2} \int_{\kappa}^{\pi} \int_{t-\pi+\xi}^{t+\pi-\xi} h(\tau, \xi) \mathrm{d} \tau \mathrm{d} \xi \\
& =\frac{1}{2} \int_{0}^{\pi} \int_{t-\pi-\xi}^{t-\pi+\xi} h(\tau, \xi) \mathrm{d} \tau \mathrm{d} \xi-\frac{1}{2} \int_{\kappa}^{\pi} \int_{t-\pi-\xi}^{t+\pi-\xi} h(\tau, \xi) \mathrm{d} \tau \mathrm{d} \xi \\
& =\frac{1}{2} \int_{0}^{\pi} \int_{-\xi}^{\xi} h(\tau, \xi) \mathrm{d} \tau \mathrm{d} \xi-\frac{1}{2} \int_{\kappa}^{\pi} \int_{-\pi}^{\pi} h(\tau, \xi) \mathrm{d} \tau \mathrm{d} \xi=: c-\chi(\kappa),
\end{aligned}
$$

where in the last line we have used the periodicity of $h(\cdot, \xi)$ and (2.4).

In order to prove that $H(t, \pi)=0, \forall t \in \mathbb{T}$, we need only to solve $\chi(\kappa)=c$. By the absolute continuity of the integral (with respect to the two-dimensional measure $\mathrm{d} \tau \mathrm{d} \xi) \chi(\kappa)$ is a continuous function. Moreover, since $h>0$ a.e. in $\Omega, \chi(0)>c>0$. Finally $\chi(\pi)=0$ and therefore, by continuity, there exists $\kappa \in(0, \pi)$ solving $\chi(\kappa)=c$.

Step 3: $H \in E$ is a weak solution of $\square H=h$, namely

$$
\int_{\Omega} \varphi_{t} H_{t}-\varphi_{x} H_{x}+\varphi h=0, \quad \forall \varphi \in C_{0}^{1}(\bar{\Omega}) .
$$

By Fubini Theorem and periodicity we get

$$
\begin{aligned}
& \int_{\Omega}\left(\varphi_{t}(t, x) \int_{0}^{\kappa} h(t-x+\xi, \xi) \mathrm{d} \xi+\varphi_{x}(t, x) \int_{0}^{\kappa} h(t-x+\xi, \xi) \mathrm{d} \xi\right) \mathrm{d} t \mathrm{~d} x \\
& =\int_{0}^{\pi} \int_{0}^{\kappa} \int_{0}^{2 \pi}\left(\varphi_{t}(t, x) h(t-x+\xi, \xi)+\varphi_{x}(t, x) h(t-x+\xi, \xi)\right) \mathrm{d} t \mathrm{~d} \xi \mathrm{d} x \\
& =\int_{0}^{\pi} \int_{0}^{\kappa} \int_{0}^{2 \pi}\left(\varphi_{t}(t+x-\xi, x)+\varphi_{x}(t+x-\xi, x)\right) h(t, \xi) \mathrm{d} t \mathrm{~d} \xi \mathrm{d} x \\
& =\int_{0}^{\kappa} \int_{0}^{2 \pi} h(t, \xi) \int_{0}^{\pi} \frac{\mathrm{d}}{\mathrm{d} x}(\varphi(t+x-\xi, x)) \mathrm{d} x \mathrm{~d} t \mathrm{~d} \xi=0
\end{aligned}
$$

by Dirichlet boundary conditions. Analogously,

$$
-\int_{\Omega}\left(\varphi_{t}(t, x) \int_{0}^{\kappa} h(t-x-\xi, \xi) \mathrm{d} \xi+\varphi_{x}(t, x) \int_{0}^{\kappa} h(t-x-\xi, \xi) \mathrm{d} \xi\right) \mathrm{d} t \mathrm{~d} x=0 .
$$


Moreover, again by Fubini Theorem,

$$
\begin{aligned}
\int_{\Omega} & \left(-\varphi_{t}(t, x) \int_{\kappa}^{x} h(t+x-\xi, \xi) \mathrm{d} \xi+\varphi_{x}(t, x) \int_{\kappa}^{x} h(t+x-\xi, \xi) \mathrm{d} \xi\right) \mathrm{d} t \mathrm{~d} x \\
& =\int_{0}^{\pi} \int_{\kappa}^{x} \int_{0}^{2 \pi}\left(-\varphi_{t}(t, x) h(t+x-\xi, \xi)+\varphi_{x}(t, x) h(t+x-\xi, \xi)\right) \mathrm{d} t \mathrm{~d} \xi \mathrm{d} x \\
= & \int_{0}^{\pi} \int_{\kappa}^{x} \int_{0}^{2 \pi}\left(-\varphi_{t}(t-x+\xi, x)+\varphi_{x}(t-x+\xi, x)\right) h(t, \xi) \mathrm{d} t \mathrm{~d} \xi \mathrm{d} x \\
= & \int_{0}^{2 \pi} \int_{0}^{\pi} h(t, \xi) \int_{\xi}^{\pi} \frac{\mathrm{d}}{\mathrm{d} x}(\varphi(t-x+\xi, x)) \mathrm{d} x \mathrm{~d} t \mathrm{~d} \xi-\int_{0}^{2 \pi} \int_{0}^{\kappa} h(t, \xi) \int_{0}^{\pi} \frac{\mathrm{d}}{\mathrm{d} x}(\varphi(t-x+\xi, x)) \mathrm{d} x \mathrm{~d} t \mathrm{~d} \xi=-\int_{\Omega} h \varphi
\end{aligned}
$$

and, analogously,

$$
\int_{\Omega}\left(\varphi_{t}(t, x) \int_{\kappa}^{x} h(t-x+\xi, \xi) \mathrm{d} \xi+\varphi_{x}(t, x) \int_{\kappa}^{x} h(t-x+\xi, \xi) \mathrm{d} \xi\right) \mathrm{d} t \mathrm{~d} x=-\int_{\Omega} h \varphi .
$$

Summing (4.86)-(4.88) and recalling (4.82), (4.83) we get (4.85).

Step 4: $H(t, x)>0$ in $\Omega$.

First case: $0<x \leqslant \kappa$. By (4.81) and geometrical considerations on the domains of the integrals, we derive that, for $0<x<\kappa, H(t, x)=\int_{\Theta} h(\tau, \xi) \mathrm{d} \tau \mathrm{d} \xi$ where $\Theta:=\Theta_{t, x}$ is the trapezoidal region in $\Omega$ with a vertex in $(\tau, \xi)=(t, x)$ and delimited by the straight lines $\tau=t-x+\xi, \tau=t+x-\xi, \xi=\kappa$ and $\tau=t-x-\xi$. Since $h>0$ a.e. in $\Omega$ we conclude that $H(t, x)>0$.

Second case: $\kappa<x<\pi$. Since $H(t+\pi-x, \pi)=0$ we have, by (4.81),

$$
\int_{0}^{\kappa} \int_{t-x-\xi}^{t-x+\xi} h(\tau, \xi) \mathrm{d} \tau \mathrm{d} \xi=\int_{\kappa}^{\pi} \int_{t-x+\xi}^{t-x-\xi+2 \pi} h(\tau, \xi) \mathrm{d} \tau \mathrm{d} \xi
$$

Therefore, substituting in (4.81), we get, for $\kappa<x<\pi$, the expression $H(t, x)=\int_{\Theta} h(\tau, \xi) \mathrm{d} \tau \mathrm{d} \xi$ where, now, $\Theta:=$ $\Theta_{t, x}$ is the trapezoidal region in $\Omega$ with a vertex in $(\tau, \xi)=(t, x)$ and delimited by the straight lines $\tau=t-x+\xi$, $\tau=t-x-\xi+2 \pi, \xi=\kappa$ and $\tau=t+x-\xi$. Since $h>0$ a.e. in $\Omega$ we conclude also in this case that $H(t, x)>0$.

Proof of Theorem 1. Since $h>0$ a.e. in $\Omega$, by Proposition 4.11 there exists a weak solution $H \in E$ of $\square H=h$ verifying (1.8) (i.e. $H>0$ in $\Omega$ ). Therefore existence of a weak solution $u \in E$ satisfying $\|u\|_{E} \leqslant C|\varepsilon|$ follows from Theorem 2(i) with $\beta(x) \equiv \beta$ and $\mathcal{R} \equiv 0$. The higher regularity for $u$ and the estimate $\|u\|_{H^{j+1}(\Omega)}+\|u\|_{C^{j}(\bar{\Omega})} \leqslant C|\varepsilon|$ follow from Theorem 2(ii) and (1.10) in Remark 1.3 since assumption (1.9) is trivially verified $(\mathcal{R} \equiv 0)$.

\section{Proof of Theorem 3}

In order to prove Theorem 3 we perform the Lyapunov-Schmidt reduction of Section 3 and we minimize the reduced action functional $\Phi$ in $\overline{B_{R}}:=\left\{\|v\|_{H^{1}} \leqslant R\right\}$. To conclude the existence of a solution, we have to prove that the minimum $\bar{v} \in \overline{B_{R}}$ is an interior minimum point in $B_{R}$ for some $R>0$.

This case is easier that the previous one since the required a-priori estimates can be deduced directly by the 0th-order variational inequality (1.17) which does not vanish for $\varepsilon=0$.

Step 1: The $L^{\infty}$-estimate. Since $a(x, u)$ satisfies (1.11) or (1.12), by (2.18), $\int_{\Omega} a(x, \bar{v}) \varphi=0, \forall \varphi \in V$ (as $\bar{v} \in V$ ) and hence

$$
\int_{\Omega} f(\bar{v}) \varphi=\int_{\Omega} \tilde{f}(\bar{v}) \varphi+a(x, \bar{v}) \varphi=\int_{\Omega} \tilde{f}(\bar{v}) \varphi .
$$


Since $\|w(\bar{v}, \varepsilon)\|_{E} \leqslant C_{0}(R)|\varepsilon|$, by the variational inequality (1.17) and (5.1), we find

$$
\int_{\Omega} \tilde{f}(\bar{v}) \varphi \leqslant \mathrm{o}(1) C_{1}\left(\|\bar{v}\|_{L^{\infty}}\right)\|\varphi\|_{L^{1}} \leqslant \mathrm{o}(1) C_{1}(R)\|\varphi\|_{L^{1}},
$$

where $C_{1}(\cdot)$ is a suitable increasing function depending on $f$. Then there exists a decreasing function $0<\varepsilon_{1}(\cdot) \leqslant \varepsilon_{0}(\cdot)$ such that

$$
\int_{\Omega} \tilde{f}(\bar{v}) \varphi \leqslant\|\varphi\|_{L^{1}} \quad \text { for }|\varepsilon| \leqslant \varepsilon_{1}(R) .
$$

We now choose, as in Subsection 4.3, the admissible variation $\varphi=q\left(\bar{v}_{+}\right)-q\left(\bar{v}_{-}\right)$where $q$ is defined in (4.20). By the mean value theorem

$$
\tilde{f}(t, x, \bar{v})=\tilde{f}(t, x, 0)+\tilde{f}_{u}(\text { intermediate point }) \bar{v}
$$

and, by (5.2), since $\tilde{f}_{u} \geqslant \beta>0$ and $\bar{v} \varphi \geqslant 0$ (recall (4.22)), we obtain

$$
\beta \int_{\Omega} \bar{v} \varphi \leqslant \kappa_{1}\|\varphi\|_{L^{1}}
$$

Arguing as at the end of Subsection 4.3 (see inequality (4.39), recall (4.31) and $M:=\|\hat{\bar{v}}\|_{L^{\infty}(\mathbb{T})} / 2$ ) we deduce

$$
\int_{\Omega} \bar{v} \varphi \geqslant \kappa_{2}\|\bar{v}\|_{L^{\infty}}\|\varphi\|_{L^{1}}
$$

and, by (5.3), we deduce

$$
\|\bar{v}\|_{L^{\infty}} \leqslant \kappa_{3} \quad \text { for }|\varepsilon| \leqslant \varepsilon_{1}(R) .
$$

Step 2: The $H^{1}$-estimate. The $H^{1}$-estimate is carried out as in Subsection 4.4 taking the admissible variation $\varphi:=-D_{-h} D_{h} \bar{v}$ in the variational inequality (1.17). By Lemma 2.6, denoting $\bar{w}:=w(\bar{v})$,

$$
0 \geqslant \int_{\Omega} f(\bar{v}+\bar{w}) \varphi=\int_{\Omega} D_{h} f(\bar{v}+\bar{w}) D_{h} \bar{v} \stackrel{h \rightarrow 0}{\longrightarrow} \int_{\Omega}\left(f_{t}(\bar{v}+\bar{w})+f_{u}(\bar{v}+\bar{w})\left(\bar{v}_{t}+\bar{w}_{t}\right)\right) \bar{v}_{t} .
$$

Using the $L^{\infty}$-estimate (5.4) for $\bar{v},\|\bar{w}\|_{E} \leqslant C_{0}(R)|\varepsilon|$ and (5.5)

$$
\int_{\Omega} f_{u}(\bar{v}+\bar{w}) \bar{v}_{t}^{2} \leqslant\left|\int_{\Omega}\left(f_{t}(\bar{v}+\bar{w})+f_{u}(\bar{v}+\bar{w}) \bar{w}_{t}\right) \bar{v}_{t}\right| \leqslant \kappa_{4}\left\|\bar{v}_{t}\right\|_{L^{2}}
$$

for $|\varepsilon| \leqslant \varepsilon_{2}(R) \leqslant \varepsilon_{1}(R)$.

Since $\tilde{f}_{u} \geqslant \beta>0, \int_{\Omega} a_{u}(x, \bar{v}) \bar{v}_{t}^{2}=0$ by (2.19), and $\|\bar{w}\|_{E} \leqslant C_{0}(R)|\varepsilon|$, we get

$$
\begin{aligned}
\int_{\Omega} f_{u}(\bar{v}+\bar{w}) \bar{v}_{t}^{2} & =\int_{\Omega} \tilde{f}_{u}(\bar{v}+\bar{w}) \bar{v}_{t}^{2}+a_{u}(x, \bar{v}+\bar{w}) \bar{v}_{t}^{2} \geqslant \int_{\Omega} \beta \bar{v}_{t}^{2}+\int_{\Omega}\left(a_{u}(x, \bar{v}+\bar{w})-a_{u}(x, \bar{v})\right) \bar{v}_{t}^{2} \\
& \geqslant \int_{\Omega} \beta \bar{v}_{t}^{2}-\mathrm{o}(1) \int_{\Omega} \bar{v}_{t}^{2} \geqslant \frac{\beta}{2} \int_{\Omega} \bar{v}_{t}^{2}
\end{aligned}
$$

for $|\varepsilon| \leqslant \varepsilon_{3}(R) \leqslant \varepsilon_{2}(R)$. From (5.6) and (5.7) we deduce

$$
\|\bar{v}\|_{H^{1}}<\kappa_{5} \quad \forall|\varepsilon| \leqslant \varepsilon_{3}(R) \text {. }
$$

Proof of Theorem 3 completed. For $R_{*}:=\kappa_{5}$ and $\varepsilon_{*}:=\varepsilon_{3}\left(R_{*}\right), \bar{v}$ is an interior point in $B_{R_{*}}$ and $\bar{u}:=\bar{v}+w(\bar{v}, \varepsilon)$ is a weak solution of (1.1)-(1.3). Regularity of the solution $\bar{u}$ is proved as in Subsection 4.5 and Theorem 3 follows. 


\section{Acknowledgements}

The authors thank A. Ambrosetti for having suggested the study of this subject and for useful discussions. They also thank P. Bolle, G. Mancini, M. Procesi for interesting comments. Part of this paper was written when the second author was visiting S.I.S.S.A. in Trieste.

\section{Appendix A}

Proof of Lemma 2.2. By the periodicity of $a(t, x)$ with respect to $t$

$$
\int_{\Omega_{\alpha}} a(t, x) \mathrm{d} t \mathrm{~d} x=\int_{\tilde{\Omega}_{\alpha}} a(t, x) \mathrm{d} t \mathrm{~d} x
$$

where $\widetilde{\Omega}_{\alpha}:=\{\alpha \pi<x<\pi(1-\alpha),-x<t<-x+2 \pi\}$. Under the change of variables $s_{+}:=t+x, s_{-}:=t-x$ the domain $\widetilde{\Omega}_{\alpha}$ transforms into the domain

$$
\left\{0<s_{+}<2 \pi, s_{+}-2 \pi(1-\alpha)<s_{-}<s_{+}-2 \pi \alpha\right\}
$$

and we get (2.9).

For $p, q \in L^{1}(\mathbb{T})$, by (2.9) we have

$$
\begin{aligned}
\int_{\Omega_{\alpha}} p(t+x) q(t-x) \mathrm{d} t \mathrm{~d} x & =\frac{1}{2} \int_{0}^{2 \pi} \mathrm{d} s_{+} p\left(s_{+}\right) \int_{-2 \pi+s_{+}+2 \alpha \pi}^{s_{+}-2 \alpha \pi} q\left(s_{-}\right) \mathrm{d} s_{-} \\
& =\frac{1}{2} \int_{0}^{2 \pi} \mathrm{d} s_{+} p\left(s_{+}\right)\left(\int_{0}^{2 \pi} q(s) \mathrm{d} s-\int_{s_{+}-2 \alpha \pi}^{s_{+}+2 \alpha \pi} q\left(s_{-}\right) \mathrm{d} s_{-}\right) \\
& =\frac{1}{2} \int_{0}^{2 \pi} p(s) \mathrm{d} s \int_{0}^{2 \pi} q(s) \mathrm{d} s-\frac{1}{2} \int_{0}^{2 \pi} \mathrm{d} s_{+} p\left(s_{+}\right) \int_{-2 \alpha \pi}^{2 \alpha \pi} q\left(s_{+}+z\right) \mathrm{d} z
\end{aligned}
$$

and we obtain (2.10) by Tonelli's Theorem (calling $s_{+}=y$ ).

Formula (2.11) follows by (2.10) setting $q \equiv 1$.

We now prove (2.12). Since the change of variables $(t, x) \mapsto(t, \pi-x)$ leaves the domain $\Omega_{\alpha}$ unchanged

$$
\int_{\Omega_{\alpha}} a(t, x) \mathrm{d} t \mathrm{~d} x=\int_{\Omega_{\alpha}} a(t, \pi-x) \mathrm{d} t \mathrm{~d} x,
$$

and, using also the periodicity of $p$,

$$
\begin{aligned}
\int_{\Omega_{\alpha}} f(p(t+x)) g(p(t-x)) \mathrm{d} t \mathrm{~d} x & =\int_{\Omega_{\alpha}} f(p(t+\pi-x)) g(p(t-\pi+x)) \mathrm{d} t \mathrm{~d} x \\
& =\int_{\alpha \pi}^{\pi-\alpha \pi} \int_{0}^{2 \pi} f(p(t+\pi-x)) g(p(t+\pi+x)) \mathrm{d} t \mathrm{~d} x \\
& =\int_{\alpha \pi}^{\pi-\alpha \pi} \int_{0}^{2 \pi} f(p(t-x)) g(p(t+x)) \mathrm{d} t \mathrm{~d} x
\end{aligned}
$$

proving (2.12).

Proof of Lemma 2.3. (2.13) follows from the equality $v^{2}=v_{+}^{2}+v_{-}^{2}-2 v_{+} v_{-},(2.11)$ (with $p=v_{+}^{2}, v_{-}^{2}$ and $\alpha=0$ ) and (2.10) (taking $p, q=\hat{v}, \alpha=0$ and recalling that $\hat{v}$ has zero average). 
(2.14) follows form (2.13) since $v_{t}(t, x)=\hat{v}^{\prime}(t+x)-\hat{v}^{\prime}(t-x)$ (and similarly for $v_{x}$ ).

Next, the first inequality of (2.15) follows from $v(t, x)=\hat{v}(t+x)-\hat{v}(t-x)$ recalling that, since $\hat{v}$ has zero average, there exist two positive measure sets in which $\hat{v} \geqslant 0$ and $\hat{v} \leqslant 0$. The second inequality of (2.15) is trivial.

We finally prove (2.16). Since $\hat{v}$ is continuous $\left(\hat{v} \in H^{1}(\mathbb{T})\right)$ there exists $\xi_{M}$ such that $\|\hat{v}\|_{L^{\infty}(\mathbb{T})}=\left|\hat{v}\left(\xi_{M}\right)\right|$. Being $\int_{0}^{2 \pi} \hat{v}=0$, there exists $\left|\xi_{0}-\xi_{M}\right| \leqslant \pi$ such that $\hat{v}\left(\xi_{0}\right)=0$. Hence

$$
\|\hat{v}\|_{L^{\infty}(\mathbb{T})}=\left|\hat{v}\left(\xi_{M}\right)\right|=\left|\int_{\xi_{0}}^{\xi_{M}} \hat{v}^{\prime}(s) \mathrm{d} s\right| \leqslant \sqrt{\pi}\left\|\hat{v}^{\prime}\right\|_{L^{2}(\mathbb{T})}=\frac{1}{\sqrt{2}}\left\|v_{t}\right\|_{L^{2}(\Omega)}
$$

by the Cauchy-Schwartz inequality and (2.14). Finally by (2.15),

$$
\|v\|_{L^{\infty}(\Omega)} \leqslant 2\|\hat{v}\|_{L^{\infty}(\mathbb{T})} \leqslant \sqrt{2}\left\|v_{t}\right\|_{L^{2}(\Omega)}=\frac{1}{\sqrt{2}}\left(\left\|v_{t}\right\|_{L^{2}(\Omega)}+\left\|v_{x}\right\|_{L^{2}(\Omega)}\right) \leqslant\|v\|_{H^{1}(\Omega)},
$$

where $\|v\|_{H^{1}(\Omega)}^{2}:=\|v\|_{L^{2}(\Omega)}^{2}+\left\|v_{x}\right\|_{L^{2}(\Omega)}^{2}+\left\|v_{t}\right\|_{L^{2}(\Omega)}^{2}$.

Proof of Lemma 2.4. By the change of variables $(t, x) \mapsto(t, \pi-x)$ and periodicity,

$$
\begin{aligned}
\int_{\Omega_{\alpha}} \varphi_{1} \cdots \varphi_{2 k+1} & =\int_{\alpha \pi}^{\pi-\alpha \pi} \int_{0}^{2 \pi} \prod_{j=1}^{2 k+1}\left(\hat{\varphi}_{j}(t+x)-\hat{\varphi}_{j}(t-x)\right) \mathrm{d} t \mathrm{~d} x \\
& =\int_{\alpha \pi}^{\pi-\alpha \pi} \int_{0}^{2 \pi} \prod_{j=1}^{2 k+1}\left(\hat{\varphi}_{j}(t+\pi-x)-\hat{\varphi}_{j}(t-\pi+x)\right) \mathrm{d} t \mathrm{~d} x \\
& =\int_{\alpha \pi}^{\pi-\alpha \pi} \int_{0}^{2 \pi} \prod_{j=1}^{2 k+1}\left(\hat{\varphi}_{j}(t-x)-\hat{\varphi}_{j}(t+x)\right) \mathrm{d} t \mathrm{~d} x \\
& =(-1)^{2 k+1} \int_{\Omega_{\alpha}} \varphi_{1} \cdots \varphi_{2 k+1},
\end{aligned}
$$

which implies (2.17).

With similar arguments we can prove (2.18) and (2.19).

Proof of Lemma 2.5. The inequality (2.20) follows by the convexity of $t \rightarrow t^{2 k}$.

We next prove (2.21). If $b=0$ it is trivially true. If $b \neq 0$ let us divide for $b^{2 k}$ and set $x:=a / b \in \mathbb{R}$. (2.21) is equivalent to prove

$$
f(x):=(x-1)^{2 k}-x^{2 k}-1+2 k x^{2 k-1}+2 k x \geqslant 0 .
$$

It results $f(0)=0, f^{\prime}(x)=2 k\left[(x-1)^{2 k-1}-x^{2 k-1}+(2 k-1) x^{2 k-2}+1\right]$ and so $f^{\prime}(0)=0$. Therefore to prove (A.1) it is sufficient to show that $f$ is convex. We have $f^{\prime \prime}(x)=2 k(2 k-1) g(x)$ where $g_{k}(x):=(x-1)^{2 k-2}-x^{2 k-2}+$ $(2 k-2) x^{2 k-3}, k \geqslant 2$.

We now show by induction on $k \geqslant 2$ that $g_{k}(x)>0$. It is true for $k=2$ since $g_{2}(x)=(x-1)^{2}-x^{2}+2 x=1>0$. Supposing now $g_{k}(x)>0$, let us prove that $g_{k+1}(x)>0$.

We claim that

$$
g_{k+1}(x)=(x-1)^{2 k}-x^{2 k}+2 k x^{2 k-1}
$$

is a strictly convex function. Indeed

$$
g_{k+1}^{\prime}(x)=2 k\left[(x-1)^{2 k-1}-x^{2 k-1}+(2 k-1) x^{2 k-2}\right] \quad \text { and } \quad g_{k+1}^{\prime \prime}(x)=2 k(2 k-1) g_{k}(x) .
$$

By the inductive hypothesis $g_{k}(x)>0$ and therefore $g_{k+1}^{\prime \prime}(x)>0$. Moreover, being

$$
g_{k+1}(x) \approx \operatorname{cost} x^{2 k-2}, \quad \lim _{x \rightarrow \pm \infty} g_{k+1}(x)=+\infty
$$


and $g_{k+1}(x)$ possesses a unique point of global minimum $\bar{x}$ that is also the unique critical point. Now it is sufficient to show that $g_{k+1}(\bar{x})>0$.

$$
g_{k}^{\prime}(\bar{x})=2 k\left[(\bar{x}-1)^{2 k-1}-\bar{x}^{2 k-1}+(2 k-1) \bar{x}^{2 k-2}\right]=0
$$

implies that $(\bar{x}-1)^{2 k-1}=\bar{x}^{2 k-1}-(2 k-1) \bar{x}^{2 k-2}$. Substituting this equality in the expression for $g_{k+1}(\bar{x})$, we have

$$
\begin{aligned}
g_{k+1}(\bar{x}) & =(\bar{x}-1)\left[\bar{x}^{2 k-1}-(2 k-1) \bar{x}^{2 k-2}\right]-\bar{x}^{2 k}+2 k \bar{x}^{2 k-1} \\
& =\bar{x}^{2 k}-\bar{x}^{2 k-1}-(2 k-1) \bar{x}^{2 k-1}+(2 k-1) \bar{x}^{2 k-2}-\bar{x}^{2 k}+2 k \bar{x}^{2 k-1} \\
& =(2 k-1) \bar{x}^{2 k-2}>0
\end{aligned}
$$

(we use that $\bar{x} \neq 0$, in fact $g_{k+1}^{\prime}(0)=-2 k \neq 0$ ).

Proof of (2.22). The case $k=1$ is trivial. For $k \geqslant 2$, we divide by $b^{2 k-1}$ and define $x:=a / b \in \mathbb{R}$. We have to prove that

$$
f(x):=(x+1)^{2 k-1}-x^{2 k-1} \geqslant 4^{1-k}, \quad \forall x \in \mathbb{R} .
$$

Since

$$
f^{\prime}(x)=(2 k-1)\left[(x+1)^{2 k-2}-x^{2 k-2}\right]=0 \quad \Longleftrightarrow \quad(x+1)^{2 k-2}=x^{2 k-2} \quad \Longleftrightarrow \quad x=-\frac{1}{2}
$$

and $f(x) \rightarrow \infty$ as $|x| \rightarrow \infty$, we conclude that $x=-1 / 2$ is the unique minimum point of $f(x)$ and therefore $f(x) \geqslant$ $f(-1 / 2)=4^{1-k}$.

Proof of Lemma 2.6. Formula (2.23) follows from

$$
\begin{aligned}
D_{h}(f g)(t, x) & =\frac{f(t+h, x) g(t+h, x)-f(t, x) g(t, x)}{h} \\
& =\frac{f(t+h, x)-f(t, x)}{h} g(t, x)+f(t+h, x) \frac{g(t+h, x)-g(t, x)}{h} .
\end{aligned}
$$

We prove (2.24) by induction. It is obvious for $m=1$. We suppose (2.24) holds for $m$ and prove it for $m+1$ : by (2.23) we have

$$
D_{h}\left(f^{m} \cdot f\right)=\left(D_{h} f^{m}\right) f+T_{h} f^{m} D_{h} f=\left(D_{h} f\right)\left[\sum_{j=0}^{m-1} f^{m-j} T_{h} f^{j}+T_{h} f^{m}\right]=\left(D_{h} f\right) \sum_{j=0}^{m} f^{(m+1)-j-1} T_{h} f^{j} .
$$

Formula (2.25) follows by (2.23) performing the change of variables $s=t+h$,

$$
\begin{aligned}
\int_{\Omega} D_{h}(f g)(t, x) & =\int_{\Omega}\left(D_{h} f\right) g+\int_{\Omega} f(t+h, x) \frac{g(t+h, x)-g(t, x)}{h} \mathrm{~d} t \mathrm{~d} x \\
& =\int_{\Omega}\left(D_{h} f\right) g+\int_{\Omega} f(s, x) \frac{g(s, x)-g(s-h, x)}{h} \mathrm{~d} s \mathrm{~d} x .
\end{aligned}
$$

We now prove formula (2.26) for integration by parts. Due to the periodicity of $f$ and $g$ with respect to $t$

$$
\begin{aligned}
\int_{\Omega} f\left(D_{-h} g\right) & =-\frac{1}{h} \int_{\Omega} f(t, x)[g(t-h, x)-g(t, x)] \mathrm{d} t \mathrm{~d} x \\
& =-\frac{1}{h} \int_{\Omega}[f(t+h, x)-f(t, x)] g(t, x) \mathrm{d} t \mathrm{~d} x=-\int_{\Omega}\left(D_{h} f\right) g .
\end{aligned}
$$

The proof of (2.27) is standard. Let $\varphi \in C^{1}(\Omega)$. By (2.26)

$$
\int_{\Omega}\left(D_{h} f\right) \varphi=-\int_{\Omega} f\left(D_{-h} \varphi\right)
$$


Now the sequence $f\left(D_{-h} \varphi\right)$ converges to $f \varphi_{t}$ a.e. and, since

$$
\left|f(t, x)\left(D_{-h} \varphi\right)(t, x)\right| \leqslant\|\varphi\|_{C^{1}(\Omega)}|f(t, x)| \in L^{1}(\Omega),
$$

we can use the Lebesgue Theorem to obtain

$$
\int_{\Omega} f\left(D_{-h} \varphi\right) \stackrel{h \rightarrow 0}{\longrightarrow} \int_{\Omega} f \varphi_{t} .
$$

Since, by hypothesis, $D_{h} f$ is bounded in $L^{2}(\Omega), D_{h} f \stackrel{L^{2}}{\rightarrow} g$, up to a subsequence. Passing to the limit in (A.2) for $h \rightarrow 0$ we find $\int_{\Omega} g \varphi=-\int_{\Omega} f \varphi_{t}$. Therefore $f$ has a weak derivative $f_{t}=g$ and by the weakly lower semicontinuity of the norm

$$
\left\|f_{t}\right\|_{L^{2}} \leqslant \liminf \left\|D_{h} f\right\|_{L^{2}} \leqslant C .
$$

In order to prove (2.28) assume temporarily $f$ is smooth. From the fundamental theorem of calculus

$$
\left(D_{h} f\right)(t, x)=\frac{f(t+h, x)-f(t, x)}{h}=\int_{0}^{1} f_{t}(t+h s, x) \mathrm{d} s .
$$

By Cauchy-Schwartz inequality, Fubini Theorem and periodicity we obtain

$$
\begin{aligned}
\int_{\Omega}\left|D_{h} f(t, x)\right|^{2} \mathrm{~d} t \mathrm{~d} x & =\int_{0}^{\pi} \int_{0}^{2 \pi}\left|\int_{0}^{1} f_{t}(t+h s, x) \mathrm{d} s\right|^{2} \mathrm{~d} t \mathrm{~d} x \leqslant \int_{0}^{\pi} \int_{0}^{2 \pi} \int_{0}^{1}\left|f_{t}(t+h s, x)\right|^{2} \mathrm{~d} s \mathrm{~d} t \mathrm{~d} x \\
& =\int_{0}^{\pi} \int_{0}^{1} \int_{0}^{2 \pi}\left|f_{t}(t+h s, x)\right|^{2} \mathrm{~d} t \mathrm{~d} s \mathrm{~d} x=\int_{0}^{\pi} \int_{0}^{1} \int_{0}^{2 \pi}\left|f_{t}(t, x)\right|^{2} \mathrm{~d} t \mathrm{~d} s \mathrm{~d} x \\
& =\int_{0}^{1} \int_{0}^{\pi} \int_{0}^{2 \pi}\left|f_{t}(t, x)\right|^{2} \mathrm{~d} t \mathrm{~d} x \mathrm{~d} s=\left\|f_{t}\right\|_{L^{2}(\Omega)}^{2} .
\end{aligned}
$$

Inequality (2.28) is valid, for any $f$ having a weak derivative $f_{t} \in L^{2}(\Omega)$, by approximation.

In order to prove (2.29) we first show the weak $L^{2}$-convergence. Let $\varphi \in C^{1}(\Omega)$. By (2.26), applying as before the Lebesgue Theorem, and since $f$ has a weak derivative $f_{t}$

$$
\int_{\Omega}\left(D_{h} f\right) \varphi=-\int_{\Omega} f\left(D_{-h} \varphi\right) \stackrel{h \rightarrow 0}{\longrightarrow}-\int_{\Omega} f \varphi_{t}=\int_{\Omega} f_{t} \varphi, \quad \forall \varphi \in C^{1}(\Omega) .
$$

Since, by (2.28), $D_{h} f$ is bounded in $L^{2}$, and (A.4) holds in the dense subset $C^{1}(\Omega) \subset L^{2}(\Omega)$, we conclude the weak $L^{2}$-convergence $D_{h} f \stackrel{L^{2}}{\rightarrow} f_{t}$.

By the weakly lower semicontinuity of the norm and (2.28)

$$
\left\|f_{t}\right\|_{L^{2}(\Omega)} \leqslant \liminf \left\|D_{h} f\right\|_{L^{2}(\Omega)} \leqslant\left\|f_{t}\right\|_{L^{2}(\Omega)} \quad \Longrightarrow \quad \lim \left\|D_{h} f\right\|_{L^{2}(\Omega)}=\left\|f_{t}\right\|_{L^{2}(\Omega)} .
$$

Since $L^{2}(\Omega)$ is a Hilbert space, weak convergence $D_{h} f \stackrel{L^{2}}{\rightarrow} f_{t}$ and (A.5) imply the strong convergence $D_{h} f \stackrel{L^{2}}{\rightarrow} f_{t}$.

Proof of (4.47). $(\Rightarrow)$ If $1 \in D$ then we choose $p_{0}:=1$ and $k:=0$. If not, there exists $p \in D, p=q_{1}^{a_{1}} \cdots q_{m}^{a_{m}}$ for $q_{j}$ prime numbers and $m, a_{j} \in \mathbb{N}^{+}$. Since, by hypothesis, $u$ is not $2 \pi / q_{j}$ periodic, $\exists n_{j} \in D$ such that $n_{j} \notin q_{j} \mathbb{N}$. In this case we choose $p_{0}:=p, p_{j}:=n_{j}, k:=m$ and we claim that the greatest common divisor $\gamma:=\operatorname{gcd}\left(p_{0}, p_{1}, \ldots, p_{k}\right)=1$. If not, since $\gamma \mid p_{0}$, there exists $j_{0} \in\{1, \ldots, k\}$ such that $q_{j_{0}} \mid \gamma$. Therefore $q_{j_{0}} \mid p_{j_{0}}=n_{j_{0}}$ which is a contradiction.

$(\Leftarrow)$ If false, there exists $n \geqslant 2$ such that $f$ is $2 \pi / n$ periodic and then $D \subseteq n \mathbb{N}$. Hence $n \mid \operatorname{gcd}\left(p_{0}, p_{1}, \ldots, p_{k}\right)=1$ which is a contradiction. 


\section{References}

[1] A. Ambrosetti, A. Malchiodi, Perturbation Methods and Semilinear Elliptic Problems on $\mathbb{R}^{n}$, Birkhäuser, in press.

[2] D. Bambusi, S. Paleari, Families of periodic solutions of resonant PDEs, J. Nonlinear Sci. 11 (1) (2001) 69-87.

[3] T. Bartsch, Y.H. Ding, C. Lee, Periodic solutions of a wave equation with concave and convex nonlinearities, J. Differential Equations 153 (1) (1999) 121-141.

[4] M. Berti, L. Biasco, Periodic solutions of nonlinear wave equations with non-monotone forcing terms, Rend. Mat. Acc. Naz. Lincei, s. 9 16 (2) (2005) 109-116.

[5] M. Berti, P. Bolle, Periodic solutions of nonlinear wave equations with general nonlinearities, Comm. Math. Phys. 243 (2) (2003) $315-328$.

[6] M. Berti, P. Bolle, Multiplicity of periodic solutions of nonlinear wave equations, Nonlinear Anal. 56 (2004) 1011-1046.

[7] M. Berti, P. Bolle, Cantor families of periodic solution for completely resonant wave equations, Preprint SISSA, 2004.

[8] J. Bourgain, Periodic solutions of nonlinear wave equations, in: Harmonic Analysis and Partial Differential Equations, in: Chicago Lectures in Math., Univ. Chicago Press, 1999, pp. 69-97.

[9] H. Brézis, J.-M. Coron, L. Nirenberg, Free vibrations for a nonlinear wave equation and a theorem of P. Rabinowitz, Comm. Pure Appl. Math. 33 (5) (1980) 667-684.

[10] H. Brézis, L. Nirenberg, Forced vibrations for a nonlinear wave equation, Comm. Pure Appl. Math. 31 (1) (1978) 1-30.

[11] J.-M. Coron, Periodic solutions of a nonlinear wave equation without assumption of monotonicity, Math. Ann. 262 (2) (1983) $273-285$.

[12] L. De Simon, H. Torelli, Soluzioni periodiche di equazioni a derivate parziali di tipo iperbolico non lineari, Rend. Sem. Mat. Univ. Padova 40 (1968) 380-401.

[13] G. Gentile, V. Mastropietro, M. Procesi, Periodic solutions for completely resonant nonlinear wave equations, Comm. Math. Phys. 256 (2005) 437-490.

[14] W.S. Hall, On the existence of periodic solutions for the equations $D_{t t} u+(-1)^{p} D_{x}^{2 p} u=\varepsilon f(\cdot, \cdot, u)$, J. Differential Equations 7 (1970) 509-526.

[15] H. Hofer, On the range of a wave operator with non-monotone nonlinearity, Math. Nachr. 106 (1982) 327-340.

[16] H. Lovicarová, Periodic solutions of a weakly nonlinear wave equation in one dimension, Czechoslovak Math. J. 19 (94) (1969) $324-342$.

[17] P.I. Plotnikov, L.N. Yungerman, Periodic solutions of a weakly nonlinear wave equation with an irrational relation of period to interval length, Differentsial'nye Uravneniya 24 (9) (1988) 1599-1607, 1654 (in Russian); Translation in Differential Equations 24 (9) (1988) 1059-1065 (1989).

[18] P. Rabinowitz, Periodic solutions of nonlinear hyperbolic partial differential equations, Comm. Pure Appl. Math. 20 (1967) 145-205.

[19] P. Rabinowitz, Time periodic solutions of nonlinear wave equations, Manuscripta Math. 5 (1971) 165-194.

[20] G. Torelli, Soluzioni periodiche dell'equazione non lineare $u_{t t}-u_{x x}+\varepsilon F(x, t, u)=0$, Rend. Istit. Mat. Univ. Trieste 1 (1969) $123-137$.

[21] M. Willem, Density of the range of potential operators, Proc. Amer. Math. Soc. 83 (2) (1981) 341-344. 